\title{
The Identification of Zinc-Finger Protein 433 as a Possible Prognostic Biomarker for Clear-Cell Renal Cell Carcinoma
}

\author{
Simone O. Heyliger ${ }^{1}$, Karam F. A. Soliman ${ }^{2}$ (D), Marilyn D. Saulsbury ${ }^{1}$ and Romonia Renee Reams ${ }^{2, *}$ \\ 1 Department of Pharmaceutical Sciences, School of Pharmacy, Hampton University, Hampton, VA 23668, USA; \\ simone.heyliger@hamptonu.edu (S.O.H.); marilyn.saulsbury@hamptonu.edu (M.D.S.) \\ 2 College of Pharmacy and Pharmaceutical Sciences, Florida A\&M University, Tallahassee, FL 32307, USA; \\ karam.soliman@famu.edu \\ * Correspondence: renee.reams@famu.edu; Tel.: +1-850-561-2672
}

Citation: Heyliger, S.O.; Soliman, K.F.A.; Saulsbury, M.D.; Reams, R.R. The Identification of Zinc-Finger Protein 433 as a Possible Prognostic Biomarker for Clear-Cell Renal Cell Carcinoma. Biomolecules 2021, 11, 1193. https://doi.org/10.3390/ biom11081193

Academic Editor: Yong-Sang Song

Received: 21 June 2021

Accepted: 6 August 2021

Published: 12 August 2021

Publisher's Note: MDPI stays neutral with regard to jurisdictional claims in published maps and institutional affiliations.

Copyright: () 2021 by the authors. Licensee MDPI, Basel, Switzerland. This article is an open access article distributed under the terms and conditions of the Creative Commons Attribution (CC BY) license (https:// creativecommons.org/licenses/by/ $4.0 /)$.
Abstract: Clear-cell renal cell carcinoma (ccRCC) is the most common and aggressive form of all urological cancers, with poor prognosis and high mortality. At late stages, ccRCC is known to be mainly resistant to chemotherapy and radiotherapy. Therefore, it is urgent and necessary to identify biomarkers that can facilitate the early detection of ccRCC in patients. In this study, the levels of transcripts of ccRCC from The Cancer Genome Atlas (TCGA) dataset were used to identify prognostic biomarkers in this disease. Analyzing the data obtained indicated that the KRAB-ZNF protein is significantly suppressed in clear-cell carcinomas. Furthermore, ZNF433 is differentially expressed in ccRCC in a stage- and histological-grade-specific manner. In addition, ZNF433 expression was correlated with metastasis, with greater node involvement associated with lower ZNF433 expression $(p<0.01)$ and with a more unsatisfactory overall survival outcome ( $\mathrm{HR}, 0.45 ; 95 \% \mathrm{CI}, 0.33-0.6 ; p=8.5 \times 10^{-8}$ ). Since ccRCC is characterized by mutations in proteins that alter epigenetic modifications and /or chromatin remodeling, we examined the expression of ZNF433 transcripts in ccRCC with wildtype and mutated forms of BAP1, KDMC5, MTOR, PBRM1, SETD2, and VHL. Analysis revealed that ZNF433 expression was significantly reduced in ccRCC with mutations in the BAP1, SETD2, and KDM5C genes $(p<0.05)$. In addition, the ZNF433 promoter region was highly methylated, and hypermethylation was significantly associated with mRNA suppression $\left(p<2.2 \times 10^{-16}\right)$. In silico analysis of potential ZNF target genes found that the largest group of target genes are involved in cellular metabolic processes, which incidentally are particularly impaired in ccRCC. It was concluded from this study that gene expression of ZNF433 is associated with cancer progression and poorer prognosis, and that ZNF433 behaves in a manner that suggests that it is a prognostic marker and a possible tumor-suppressor gene in clear-cell renal cell carcinoma.

Keywords: KRAB; zinc-finger; tumor microenvironment; BAP1; SETD2; clear cell renal carcinoma; immune response; transcription; ZNF433

\section{Introduction}

Renal cell carcinoma (RCC) is the most frequent malignancy in the kidneys, accounting for $75 \%$ of all kidney cancers and $2-3 \%$ of all cancers [1]. Clear-cell renal cell carcinoma (ccRCC) is the most common pathological subtype of RCC, originating from the proximal uriniferous tubules, and often presents an aggressive phenotype [2]. ccRCC is rarely detected early and is largely asymptomatic, and the clinicopathological risk factors cannot fully distinguish ccRCC patients [3]. At late stages, ccRCC has high morbidity and mortality and a poor prognosis, and is relatively insensitive to chemotherapy and radiotherapy [4] Therefore, it is urgent and necessary to mine kidney-cancer datasets to identify potential biomarkers that can facilitate the early detection of ccRCC in patients.

Zinc-finger proteins act as transcription regulators, and some are differentially expressed in cancers [5]. These transcription factors are structurally diverse and have been shown to mediate numerous cellular processes, such as replication and repair, transcription 
and translation, metabolism and signaling, cell proliferation, and apoptosis [6]. KRAB-ZNF proteins makeup approximately one-third of zinc-finger proteins and are probably the largest group of transcriptional regulators in mammals $[7,8]$. Despite the ubiquitous nature of these proteins, relatively little is known about the function of the KRAB-ZNF individual members. Members of the KRAB-containing protein family contain at least one KRAB domain on the $\mathrm{N}$-terminus and bind DNA through their $\mathrm{C} 2 \mathrm{H} 2$ zinc-finger domains [8]. The zinc ion interacts with two cysteine and two histidine residues to create a stabilized folded structure that consists of two or three beta-strands and one alpha-helix $[9,10]$. The alphahelix mediates DNA binding through non-covalent interactions within the DNA major groove; however, variations in the amino acid sequence of the finger domains and spacing, as well as in zinc-finger number and higher-order structure, may increase the ability to bind multiple different ligands such as RNA, DNA-RNA hybrids, and even proteins $[5,9,10]$. Nonetheless, KRAB-ZNF proteins are often tissue-specific, and are primarily thought to act as transcriptional repressors and/or epigenetic suppressors of gene expression [8,10-12].

Because of the sheer number of KRAB-ZNF proteins, their roles in oncogenesis have not been fully elucidated. ZNF471 was shown to be downregulated in esophageal squamous cell carcinoma (ESCC) cells, and restoration of expression leads to the induction of apoptosis and G0/G1 arrest, as well as inhibition of tumor cell colony formation [8]. Similarly, ZNF382 is downregulated in multiple cancer types, including leukemia and the solid tumors of the head and neck, lung, esophagus, colon, stomach, cervix, and breast, and serve as a tumor suppressor [8,13-15]. ZNF671 was found to be downregulated in brain, breast, lung, head, neck, and skin cancers, and overexpression inhibited the epithelial-mesenchyme transition (EMT), as well as cancer cell proliferation, migration, and invasion [16,17]. Taken collectively, KRAB-ZNF proteins may serve as tissue-specific tumor-suppressor genes.

Here, we report that the ZNF433, a KRAB zinc-finger transcription family member, is downregulated in clear-cell renal cell carcinoma, and its expression is reduced in a stage-specific manner. Moreover, lower expression is associated with higher tumor grades and poor overall survival.

\section{Materials and Methods}

\subsection{Gene and Protein Expression Analysis}

\subsubsection{UALCAN Analysis}

In this study, UALCAN (http://ualcan.path.uab.edu/analysis.html (accessed on 5 May 2021)) was utilized to assess the levels of transcripts in clear-cell renal cell carcinomas from the TCGA dataset. UALCAN is an online, open-access platform that contains TCGA raw data, including gene expression, protein expression (CPTAC dataset), promoter methylation, miRNA expression, and clinicopathological data [18]. UALCAN allows users to query the gene of interest and compare its expression to clinicopathological features. Specifically, UALCAN was employed in our study to analyze the mRNA expression of ZNF433 in relation to the clinicopathological parameters (cancer stage, renal cancer subtypes, metastasis, and grade) of ccRCC.

\subsubsection{TNM Plotter}

The TNM plotter (https:/ / www.tnmplot.com (accessed on 8 May 2021)) is a web application that enables a real-time comparison of gene-expression changes between tumor, normal, and metastatic tissues amongst different types of platforms across all genes. The software was used to assess ZNF433 expression using the TCGA datasets-a direct comparison between tumor and normal tissues was made by running the Mann-Whitney test [19]. 


\subsubsection{TIMER2.0}

TIMER2.0 was used to assess gene associations and co-expression patterns of genes across TCGA cancer types. It was also used to evaluate ZNF433 expression in ccRCC with wildtype and mutant forms of key transcriptional factors associated with ccRCC tumor initiation and progression. TIMER2.0 (http:/ / timer.cistrome.org, accessed on 8 May 2021) provides a robust estimation of immune infiltration levels for The Cancer Genome Atlas (TCGA) or user-provided tumor profiles using six state-of-the-art algorithms [20,21]. In addition, TIMER2.0 provides modules for investigating the associations between immune infiltrates and genetic or clinical features, clinical outcomes, and exploring cancer-related associations in the TCGA cohorts [20,21]. All TCGA tumor data used in TIMER2.0, including transcriptome profiles, somatic mutation calls, somatic copy number variations, and patient clinical outcomes, were collected from the GDAC firehose website [20,21].

\subsubsection{Human Protein Atlas}

The Human Protein Atlas (HPA) was used to ascertain whether the ZNF433 protein was differentially expressed in normal kidney and renal carcinomas (https: / / www. proteinatlas.org, accessed 14 May 2021) [22].

\subsection{Overall Survival Analysis}

The online Kaplan-Meier plotter program (https:// kmplot.com/analysis, accessed on 7 May 2021) was used to determine the prognostic value of ZNF433 expression in clear-cell renal cell carcinoma. Specifically, Kaplan-Meier survival plots were generated to ascertain whether there was a correlation between ZNF433 expression and the overall survival (OS) of clear-cell renal cell carcinoma patients [23,24]. To assess the prognostic value of ZNF433, each percentile (of expression) between the lower and upper quartiles was computed, and the best-performing threshold was used as the final cutoff in a univariate Cox regression analysis. The Kaplan-Meier survival plot and the hazard ratio with 95\% confidence intervals and logrank $p$ value were calculated and plotted in $\mathrm{R}$ using the "survplot" function of the "survival" Bioconductor package [23,24].

\subsection{Methylation Analysis}

Methylation status was assessed using MethSurv (https://biit.cs.ut.ee/methsurv, accessed on 9 May 2021). This web-based tool enables researchers to perform survival analysis for a CPG located in or around the proximity of a query gene using the TGCA dataset [25]. To ascertain if methylation status was associated with gene expression, we utilized the Shiny Methylation Analysis Resource Tool (SMART) web-based program. The SMART application integrates multiomics and clinical data with DNA methylation. It provides key interactive and customized functions, including CpG visualization, pancancer methylation profile, differential methylation analysis, correlation analysis, and survival analysis [26].

\subsection{Gene Ontology and Pathway Analysis}

The gene ontology (GO) enrichment analysis results of the genes co-expressed with ZNF433 was visualized using LinkedOmics (http:/ / www.linkedomics.org, accessed on 9 July 2021). The LinkedOmics database contains multiomics data and clinical data for 32 cancer types comprising 11,158 patients from TCGA [27]. The "LinkFinder" module was used to investigate differentially expressed genes within the TCGA KIRC cohort. The "LinkInterpreter" module was used to perform GSEA pathway enrichment analysis using the GO Biological Process and KEGG databases. Results were analyzed for significance using the Pearson's correlation test, with a $p$-value and false discovery rate (FDR or $q$-value) set at 0.05 . 
To determine if there were genes that were putative targets of ZNF433 transcription factors, we queried the Molecular Signatures Database (MSigDB) (https:/ /www.gsea-msigdb. $\mathrm{org}$ /gsea/msigdb, accessed on 9 May 2021), which is a collection of annotated genes that can be used with the Gene Set Enrichment analysis software. The predicted target genes of ZNF433 (Table 1) were then uploaded into the PANTHER (Protein ANalysis THrough Evolutionary Relationships) Classification System analysis web to identify gene/protein networks that may be over-represented within the gene set. The PANTHER website (http:/ / pantherdb.org/about.jsp, accessed on 9 May 2021) provides tools for functional analysis of lists of genes or proteins. For example, gene lists can be analyzed graphically in terms of sortable functional classes and pie or bar charts; or analyzed statistically by overrepresentation or enrichment tests [28-31].

Table 1. Clinicopathological features of the ccRCC TCGA dataset. A $t$-test was conducted to analyze differential gene expression of ZNF433 between genders. ANOVA was used to analyze ZNF433 gene expression across clinicopathological features. Statistical significance was set at $p<0.05$. N.S. $=$ not significant $(p>0.05)$.

\begin{tabular}{|c|c|c|}
\hline Clinicopathological Features & $\mathbf{N}$ & Statistics \\
\hline Gender & & N.S. \\
\hline Male & 385 & \\
\hline Female & 188 & \\
\hline Histological Grade & & $p<0.001$ \\
\hline Normal & 72 & \\
\hline 1 & 14 & \\
\hline 2 & 229 & \\
\hline 3 & 206 & \\
\hline 4 & 76 & \\
\hline Pathological Stage & & $p<0.001$ \\
\hline Normal & 72 & \\
\hline 1 & 267 & \\
\hline 2 & 57 & \\
\hline 3 & 123 & \\
\hline 4 & 84 & \\
\hline Metastasis & & $p<0.001$ \\
\hline Normal & 72 & \\
\hline N0 & 240 & \\
\hline N1 & 16 & \\
\hline Age & & N.S. \\
\hline $21-40$ years & 26 & \\
\hline $41-60$ years & 238 & \\
\hline $61-80$ years & 246 & \\
\hline $81+$ years & 23 & \\
\hline
\end{tabular}

\subsection{Statistical Analysis}

Statistical analysis of tumor and normal-tissue gene expression was conducted using the TNM plotter web analysis tool. Comparison of the normal and tumor samples was performed using a Mann-Whitney U test. Statistical significance was set at $p<0.05$. In addition, the Kaplan-Meier survival plots with the number at risk, hazard ratio (HR), $95 \%$ confidence intervals (CI), and log-rank $p$-values were obtained using the KaplanMeier plotter web analysis tool. The $p$-value was set at $p<0.05$ to indicate a statistically significant difference in overall survival in the high- and low-expression groups. For analysis of clinicopathological features, a T-test was conducted to analyze differential gene expression of ZNF433 between genders. One-way ANOVA was used to analyze ZNF433 gene expression across clinicopathological features. ROC, as well as Cox univariate and multivariate proportional hazard model analyses were performed using MedCalc software. Statistical significance was set at $p<0.05$. 


\section{Results}

\subsection{ZNF433 mRNA and Protein Expression in Normal and Clear-Cell Renal Cell Carcinoma}

Table 1 provides the types of clinicopathological features, the corresponding numbers of each phenotype that were contained in the TCGA KIRC dataset, and the significance of clinicopathological features relevant to differential expression of ZNF433.

Figure 1a depicts the expression of ZNF433 transcripts in the clear-cell renal cell carcinoma TCGA dataset. ZNF433 mRNA expression was significantly lower $\left(p=1.89 \times 10^{-20}\right)$ than in normal tissues. We examined Human Protein Atlas (https:/ /www.proteinatlas. org/, accessed on 14 May 2021) immunohistochemistry images of human normal and renal carcinoma tissues stained with antibodies raised against the ZNF433 protein (Figure 1b) to validate the gene-expression data. The renal carcinoma images showed lower ZNF433 protein expression, as documented by the descriptions accompanying each image (https: / / www.proteinatlas.org/ENSG00000197647-ZNF433/pathology/renal+cancer\#img, accessed on 14 May 2021). Renal cancer tissues had "low" or "not detected" staining with "weak" intensities, whereas all normal tissues were described as having "medium" staining with "moderate" intensities (Figure 1b), thereby suggesting that the ZNF433 protein is downregulated in renal carcinomas.

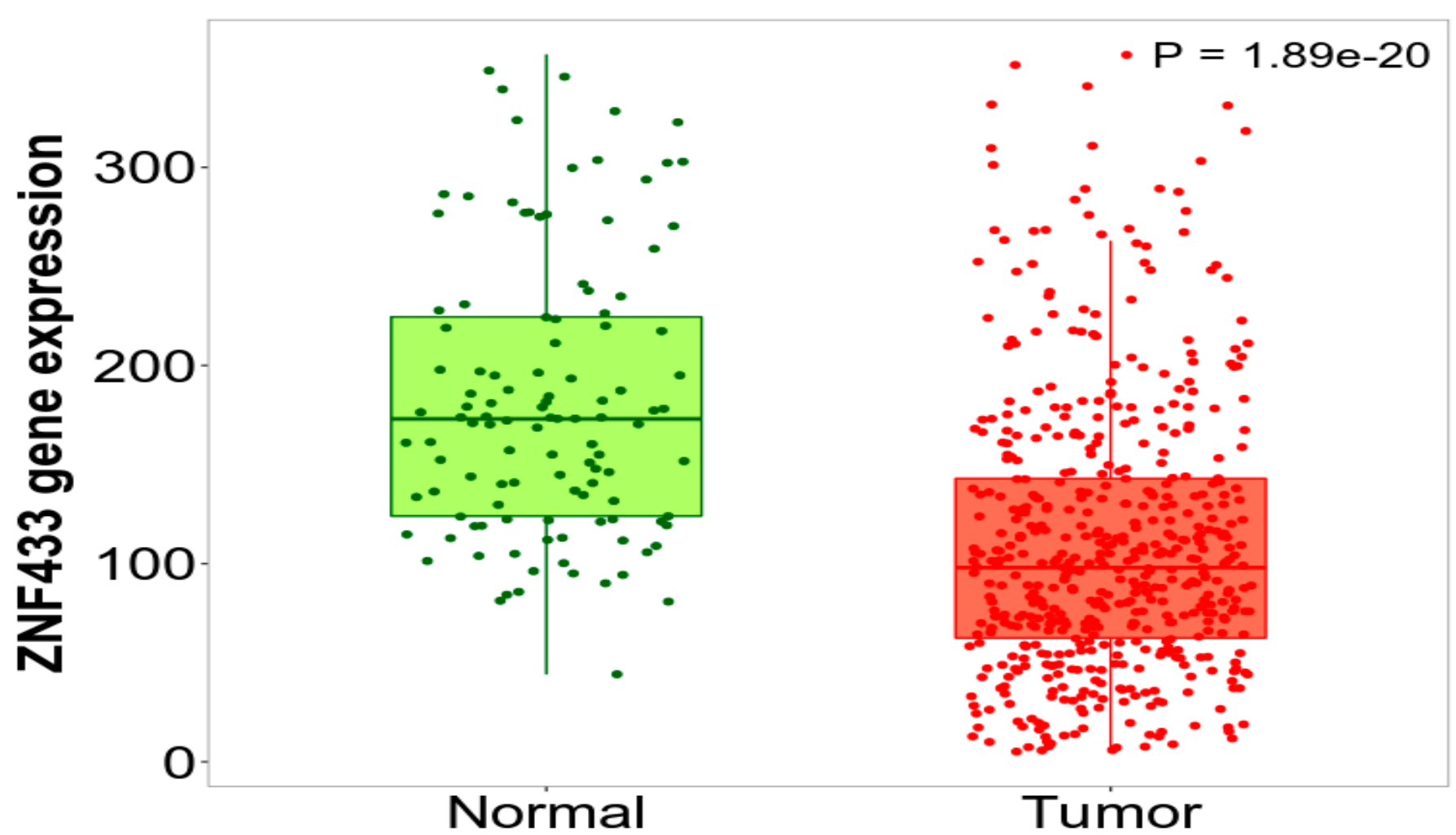

(a)

Figure 1. Cont. 


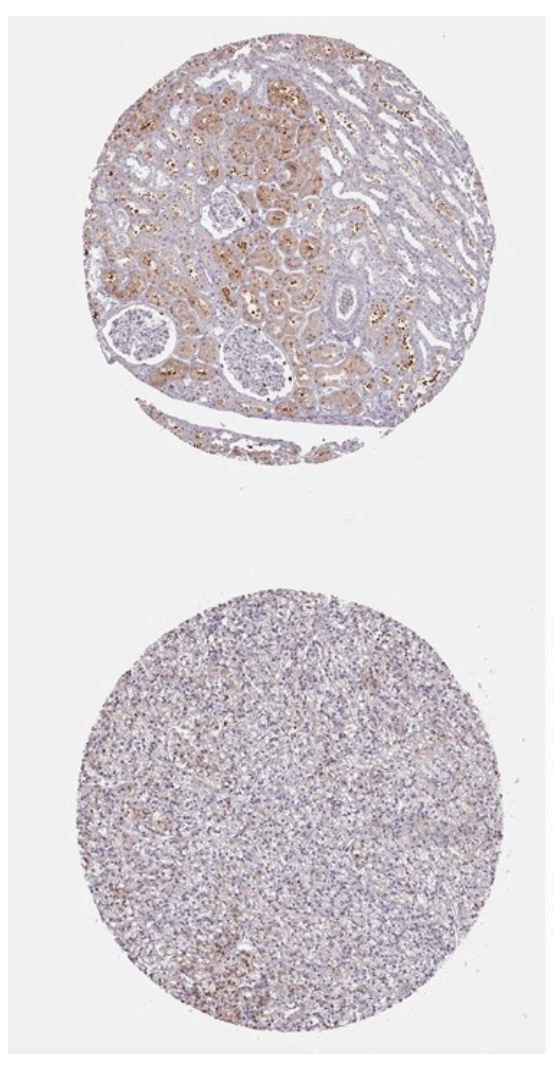

\section{ZNF433}

Female, age 41

Kidney (T-71000)

Normal tissue

Staining: Medium

Intensity: Moderate

Quantity: $75 \%-25 \%$

Location: Cytoplasmic/

membranous nuclear

Antibody: HPA073724

\section{ZNF433}

Female, age 59

Kidney (T-71000)

Adenocarcinoma

Staining: Low

Intensity: Weak

Quantity: $75 \%-25 \%$

Location: Cytoplasmic

membranous nuclear

Antibody: HPA073724

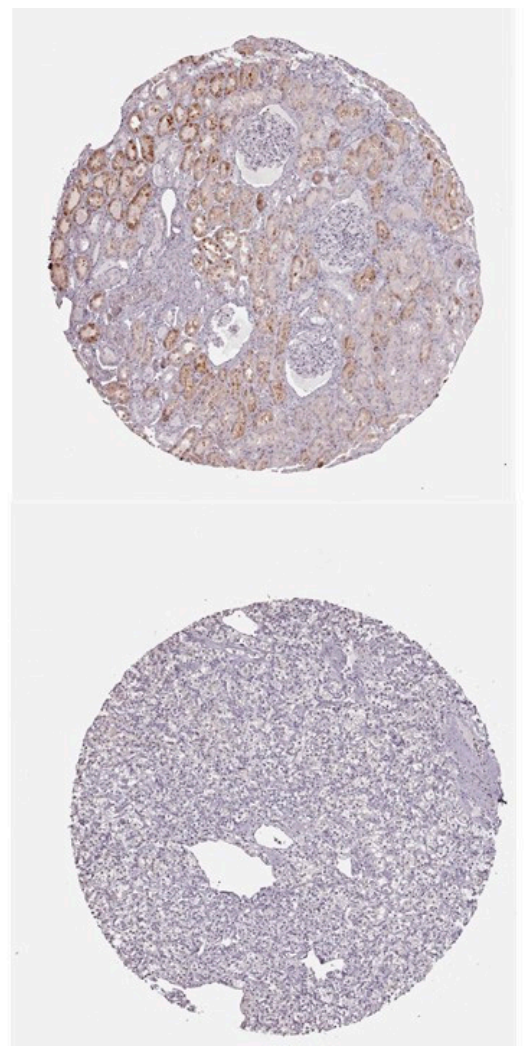

ZNF433

Male, age 73

Kidney (T-71000)

Normal tissue

Staining: Medium

Intensity: Moderate

Quantity:75\%-25\%

Location: Cytoplasmic/

membranous nuclear

Antibody: HPA073724

ZNF433

Male, age 77

Kidney (T-71000)

Adenocarcinoma

Staining: Not detected

Intensity: Weak

Quantity: None

Location: None

Antibody: HPA073724

(b)

Figure 1. Expression of ZNF433 transcripts and protein in clear-cell renal cell carcinoma. (a) Plots were generated using TNMplot analysis (https://www.tnmplot.com, 14 May 2021) of RNAseq data using TCGA datasets. ZNF433 transcripts were significantly downregulated (fold change $=0.65)$ in tumors $(n=535)$ relative to normal tissues $(n=117)$. (b) Immunohistochemistry from the Human Protein Atlas website (https:/ / www.proteinatlas.org, accessed on 14 May 2021) demonstrating expression of ZNF433 protein in normal and renal cancer tissues. The images are representative of normal kidney and renal adenocarcinoma tissues stained with anti-ZNF433 antibody (HPA073724). Representative images for tumors can be obtained at https: / / www.proteinatlas.org/ENSG00000197647-ZNF433/pathology/renal + cancer\#img (accessed on 14 May 2021), and representative images for normal kidney tissue can be found at https:/ / www.proteinatlas. org/ENSG00000197647-ZNF433/tissue/kidney (accessed on 14 May 2021).

\subsection{ZNF433 mRNA Expression in Association with the Clinicopathological Features of Clear-Cell Renal Cell Carcinoma}

To characterize ZNF433, we examined ZNF433 expression in different clinicopathological features of clear-cell renal cell carcinoma. ZNF433 remained suppressed $(p<0.001)$ across tumor stages (Figure 2a), with stages 3 and 4 having a lower expression of transcripts relative to normal as well as stages 1 and 2. A similar pattern was also noted regarding histological grades whereby ZNF433 expression was significantly downregulated in higher grades ( 3 and 4$)$ (Figure $2 b),(p<0.01)$. In addition, significant downregulation of ZNF433 transcripts (Figure 2c) was noted across metastasis status, with ZNF433 expression decreasing significantly with more nodal involvement $(p<0.01)$ and thus higher metastatic status. Finally, ZNF433 expression was suppressed in both renal carcinoma subtypes $(p<0.001)$, with the more aggressive subtype $\mathrm{ccB}$ exhibiting the lowest level of mRNA expression (Figure 2d). 
Expression of ZNF433 in KIRC based on individual cancer stages

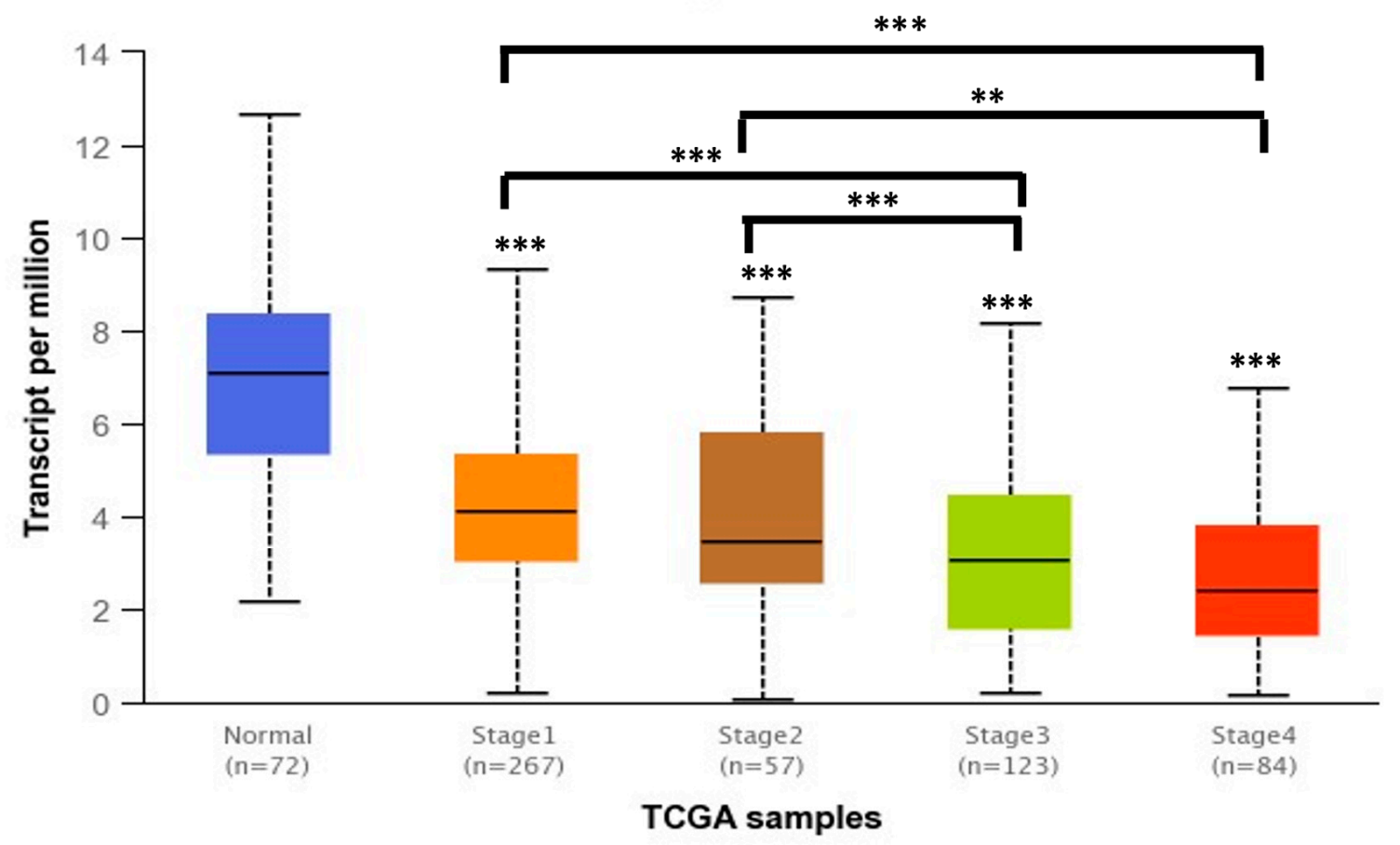

(a)

Expression of ZNF433 in KIRC based on tumor grade

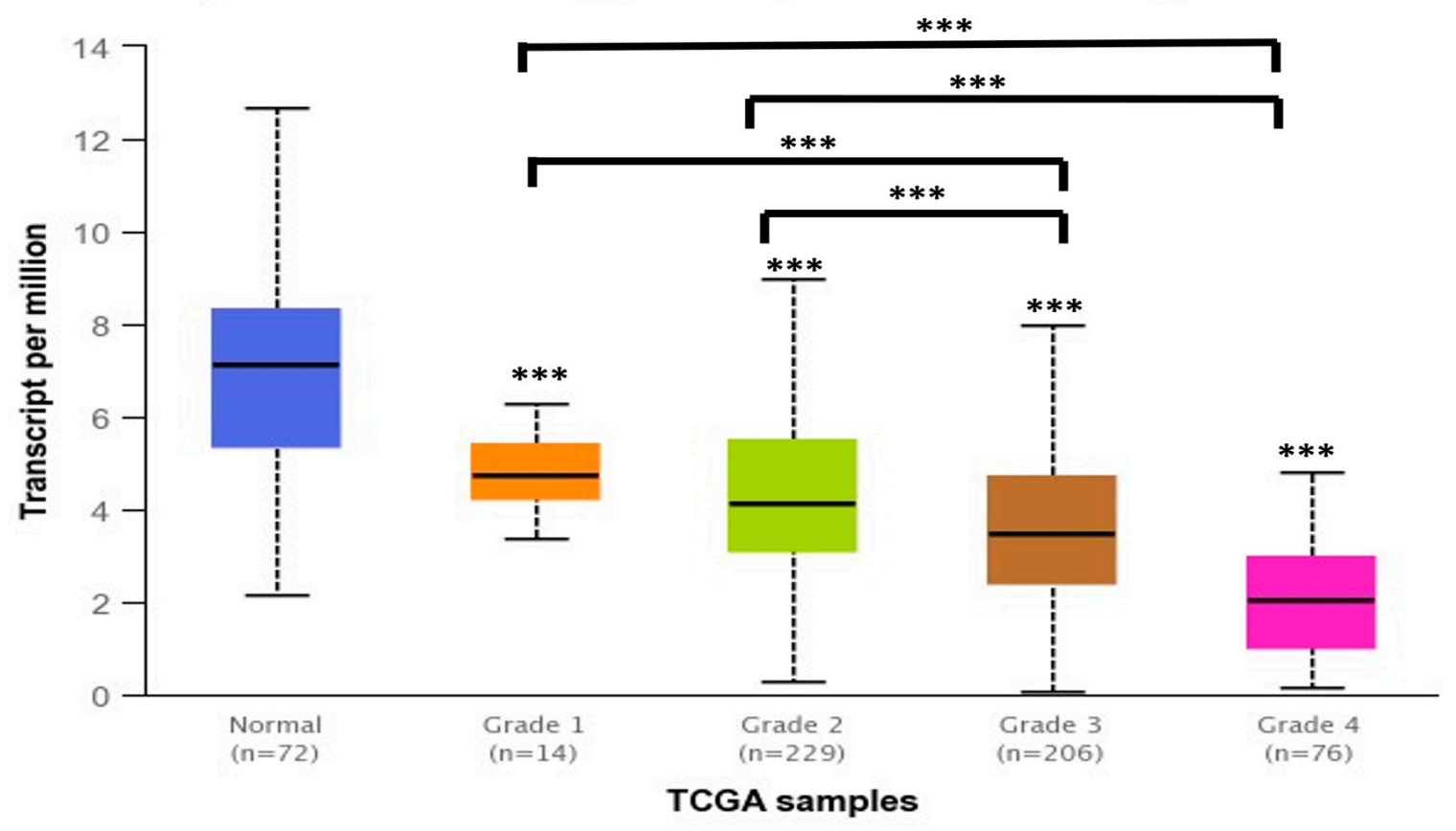

(b)

Figure 2. Cont. 


\section{Expression of ZNF433 in KIRC based on nodal metastasis status}

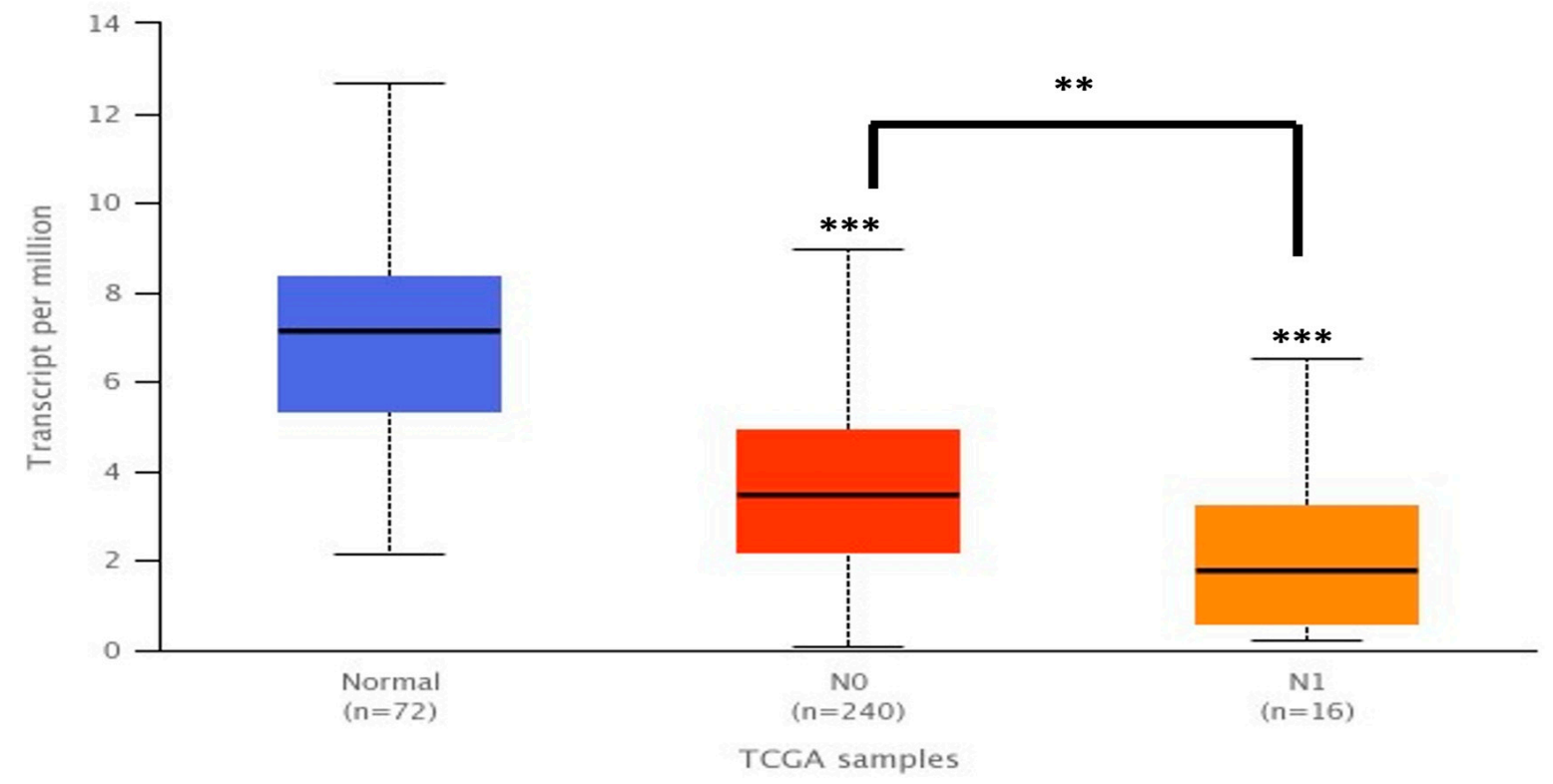

(c)

\section{Expression of ZNF433 in KIRC based on KIRC subtypes}

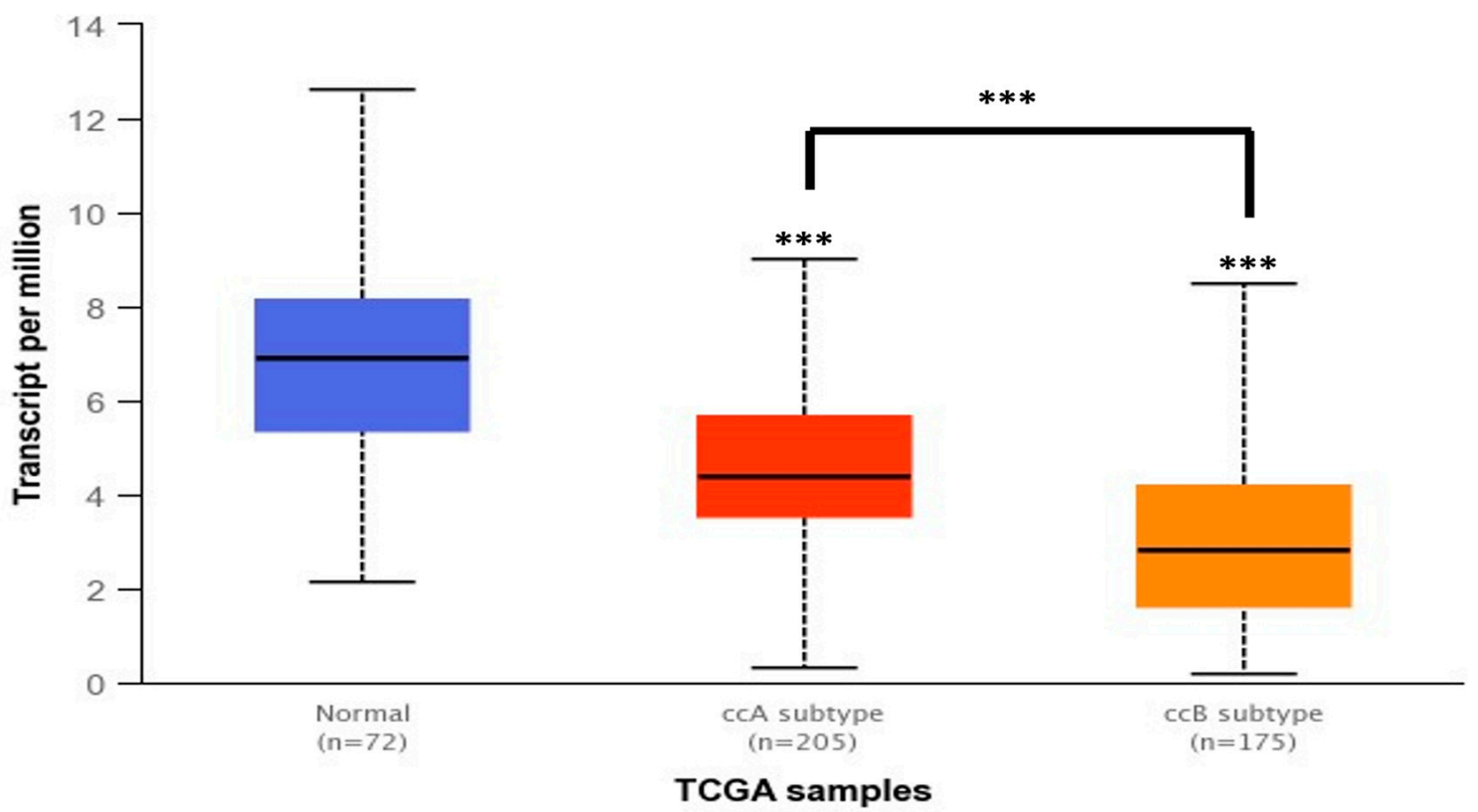

(d)

Figure 2. (a) Expression of ZNF433 transcripts across cancer stages in clear-cell renal cell carcinoma. ZNF433 transcripts were significantly downregulated in all tumor stages relative to normal tissues. (b) Expression of ZNF433 transcripts across histological grades of clear-cell renal carcinoma. ZNF433 transcripts were significantly downregulated in all tumor grades relative to normal tissues. (c) Expression of ZNF433 transcripts in metastatic tissues. ZNF433 transcripts were decreased with greater nodal involvement $(p<0.01)$. (d) Expression of ZNF433 transcripts in clear-cell renal cell carcinoma subtypes. ZNF433 transcripts were reduced in both subtypes, with ccB ccRCC exhibiting the lowest expression $(p<0.001)$. All plots were generated using UALCAN analysis of RNAseq data using TCGA datasets. ${ }^{* *} p<0.01$ and ${ }^{* * *} p<0.001$. 


\subsection{Overall Survival of Patients with Clear-Cell and Papillary Renal Cell Carcinomas as a} Function of ZNF433 Expression

A Kaplan-Meier survival analysis was performed to ascertain whether lower expression of ZNF433 is linked to changes in overall patient survival (Figure 3a). Lower expression of ZNF433 mRNA was associated with a poorer prognosis in patients with clear-cell renal cell carcinomas (HR, $0.45 ; 95 \% \mathrm{CI}, 0.33-0.6 ; p=8.5 \times 10^{-8}$ ). The median survival for the high-expression group was 118.47 months, compared to 54.2 months for the low-expression group. A receiver operating characteristic (ROC) analysis was performed to validate KM survival observations and to ascertain if ZNF433 expression could discriminate between high-expression and low-expression groups in relation to survival. The AUC was 0.701 , sensitivity $=0.66$, specificity $=0.66$, and $95 \% C I=0.67-0.729$ with $p<0.001$. We performed Cox univariate and multivariate analyses on ZNF433 expression relative clinicopathological features (Table 2). In the univariate analysis, ZNF433 expression and histological grade, stage, and age were found to be predictive of overall survival. However, only age and stage retained significance in multivariate models. The ZNF433 $p$-value was 0.056 , suggesting that there was an important tendency with regard to ZNF433 acting as a possible prognostic factor.

\section{ZNF433}

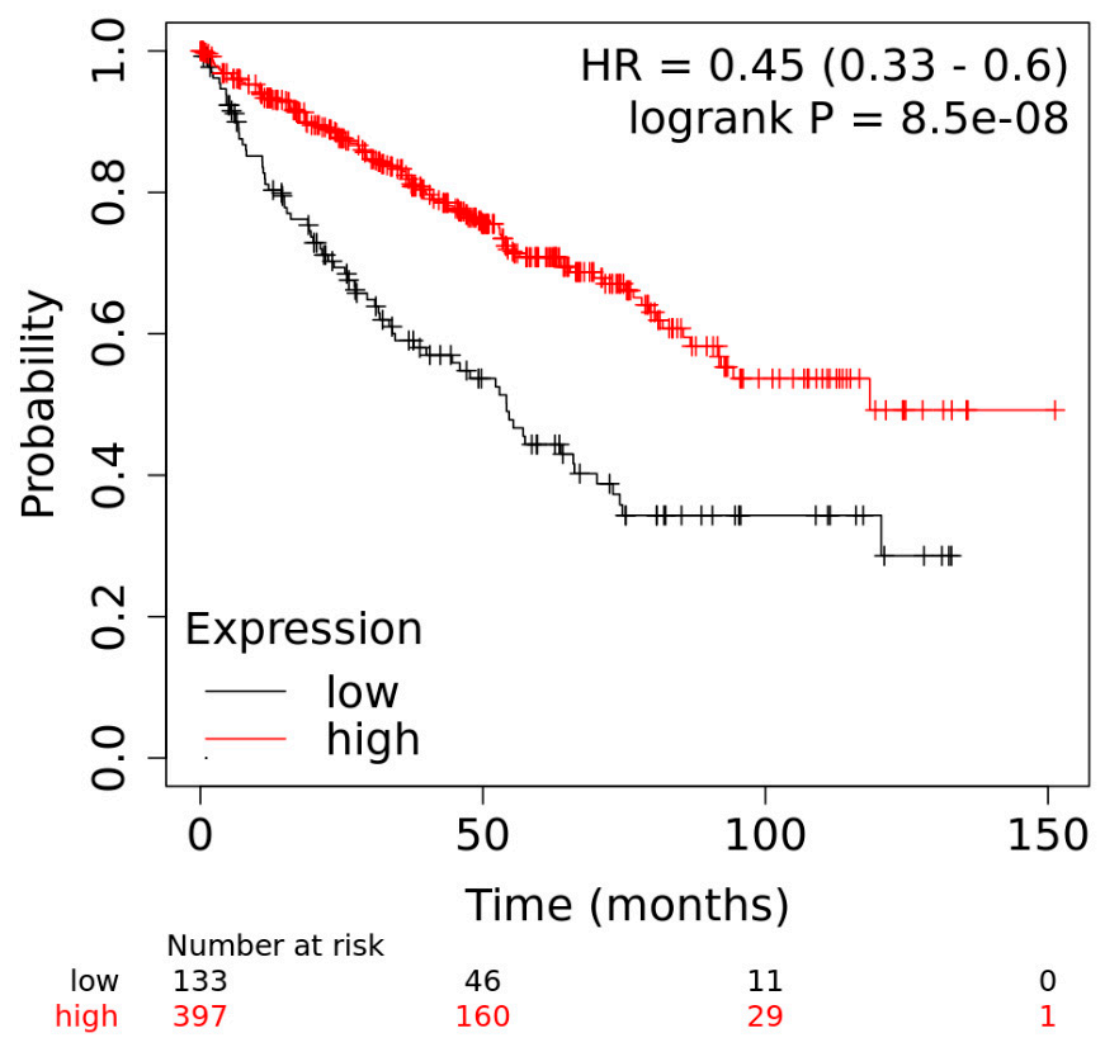

(a)

Figure 3. Cont. 


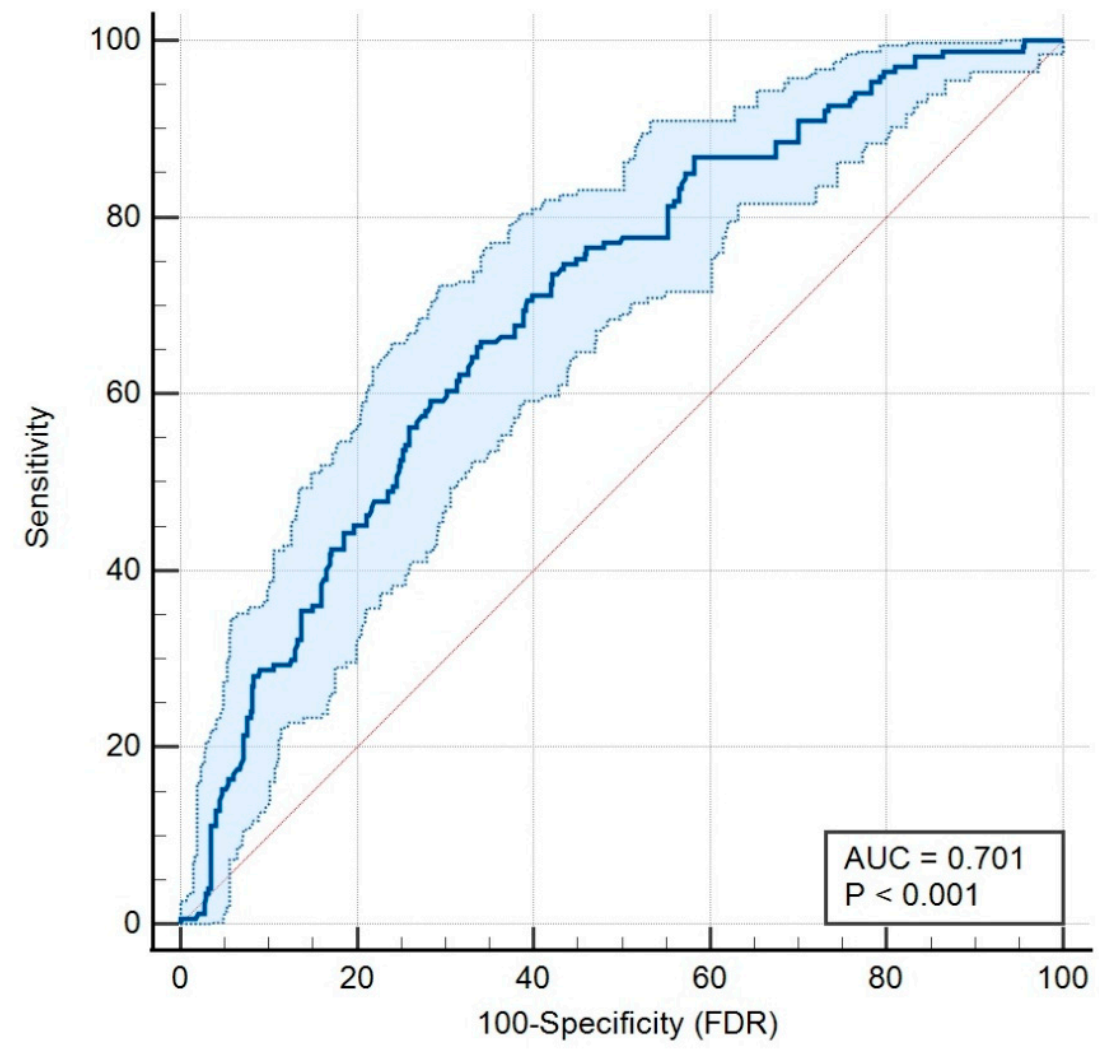

(b)

Figure 3. (a) Overall survival of patients with clear-cell and papillary renal cell carcinomas as a function of ZNF433 expression. The Kaplan-Meier survival plots were generated using K-M plotter analysis (https: / / kmplot.com/analysis, accessed on 7 May 2021) of RNAseq data from the clear-cell renal cell carcinoma $(n=533)$ TCGA datasets. The median survival for the high-expression group was 118.47 months, compared to 54.2 months for the low-expression group. (b) Receiver operating characteristic (ROC) curve of overall survival times of ZNF433 low- and high-expression patients. The ROC analysis was performed to validate KM survival observations and to ascertain if ZNF433 expression could discriminate between high-expression and low-expression groups in relation to survival. The AUC was 0.701 , sensitivity $=0.66$, specificity $=0.66$, and $95 \% \mathrm{CI}=0.67-0.729$ with $p<0.001$.

Table 2. Univariate and multivariant Cox regression analysis of age, gender, pathological stage, histological grade in relation to overall survival in ccRCC patients. The statistical significance was set at $p<0.05$.

\begin{tabular}{|c|c|c|}
\hline Clinicopathological Feature & $\begin{array}{c}\text { Univariate Analysis } \\
\text { HR }(95 \% \mathrm{CI}) \\
p \text {-Value }\end{array}$ & $\begin{array}{c}\text { Multivariate Analysis } \\
\text { HR (95\% CI) } \\
p \text {-Value }\end{array}$ \\
\hline ZNF433 & $\begin{array}{c}0.6227(0.4593-0.8440) \\
p=0.0023\end{array}$ & $\begin{array}{c}0.7390(0.6987-1.3065) \\
p=0.0597\end{array}$ \\
\hline Gender & $\begin{array}{c}0.9508(0.7003-1.2910) \\
p=0.7466\end{array}$ & $\begin{array}{c}0.9711(0.7094-1.3292) \\
p=0.8547\end{array}$ \\
\hline Age & $\begin{array}{c}1.7589(1.2979-2.3835) \\
p=0.0003\end{array}$ & $\begin{array}{c}1.5889(1.1612-2.1742) \\
p=0.0038\end{array}$ \\
\hline Stage & $\begin{array}{c}3.8179(2.7869-5.2303) \\
p<0.0001\end{array}$ & $\begin{array}{c}3.3766(2.4441-4.6649) \\
p<0.001\end{array}$ \\
\hline Grade & $\begin{array}{c}1.5172(1.1152-2.0639) \\
p=0.0079\end{array}$ & $\begin{array}{c}1.2429(0.9088-1.6697) \\
p=0.1734\end{array}$ \\
\hline
\end{tabular}




\subsection{Effect of CpG Methylation on ZNF433 Gene Expression}

Given that zinc-finger transcription factors are highly regulated by methylation, we examined the methylation status of ZNF433 and compared it relative to mRNA expression (Figure $4 \mathrm{a}, \mathrm{b})$. The ZNF433 promoter region was highly methylated compared to the control $\left(p=1.62 \times 10^{-12}\right)$, and hypermethylation correlated with lower mRNA expression $(\mathrm{R}=-0.58$; $\left.p<2.2 \times 10^{-16}\right)$. Moreover, hypermethylation at promoter sites cg01404518 and cg18566819 sites was associated with significantly lower overall survival $(p<0.05)$ (Figure $4 \mathrm{c}-\mathrm{j}$ ).

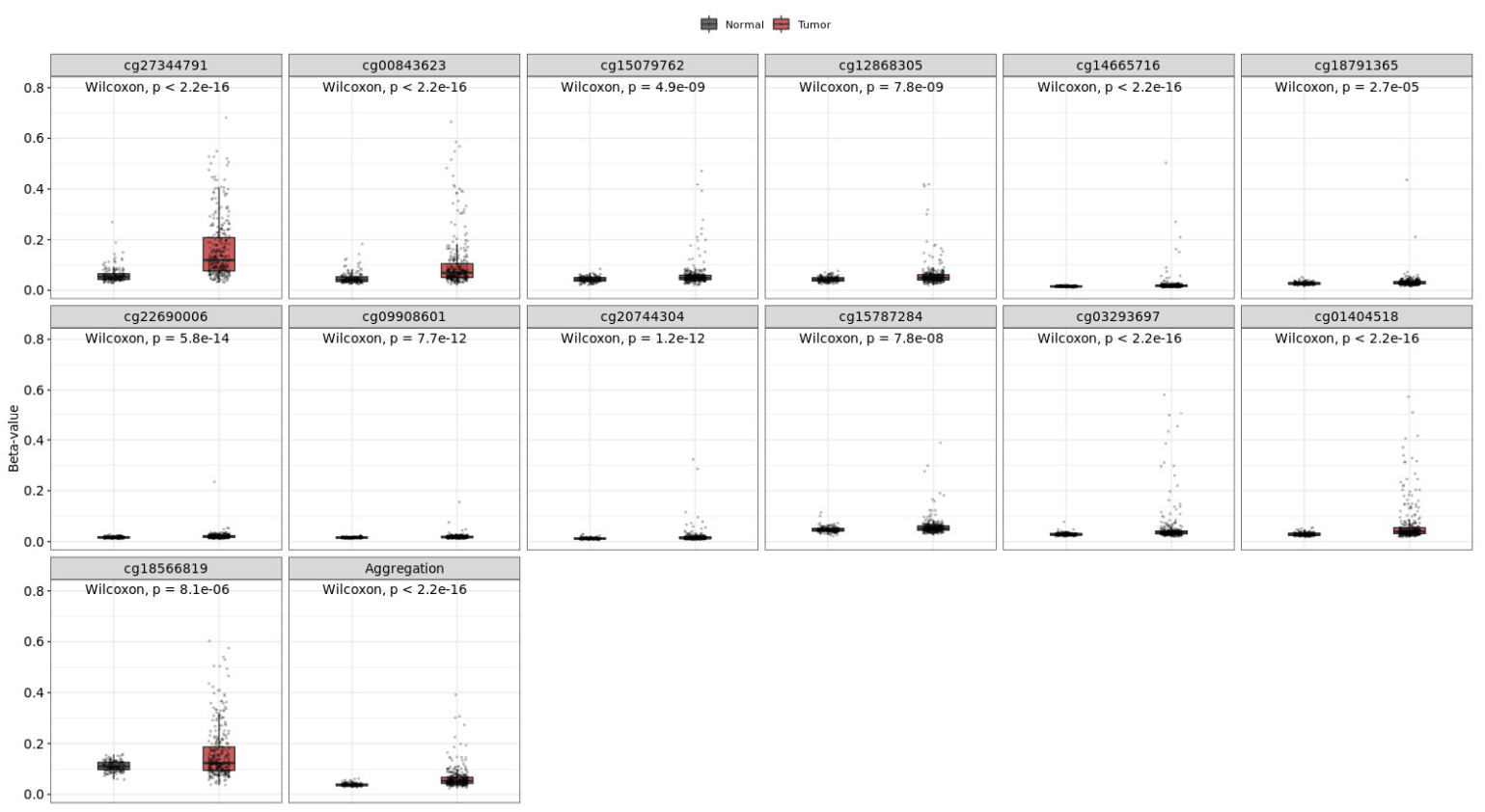

(a)

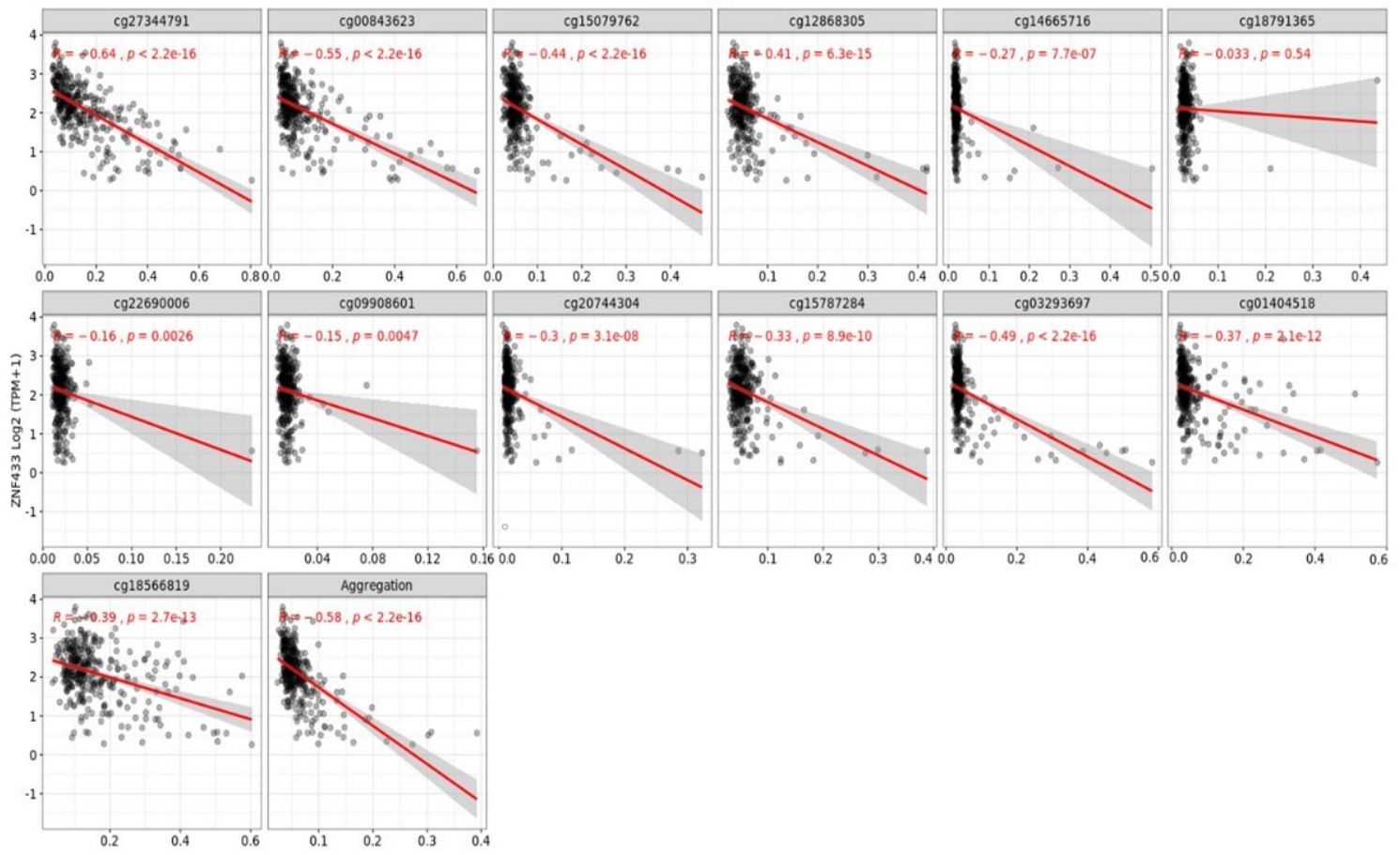

(b)

Figure 4. Cont. 


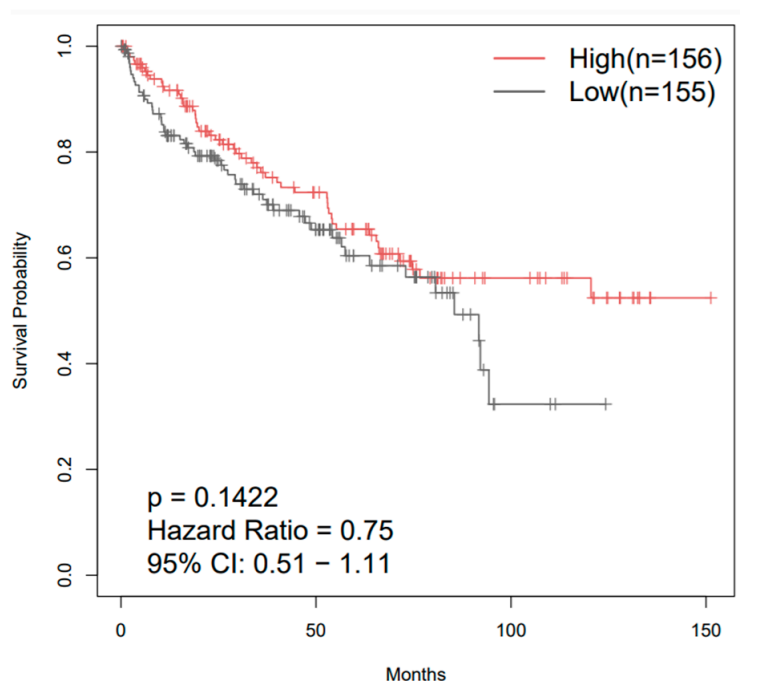

cg20744304

(c)

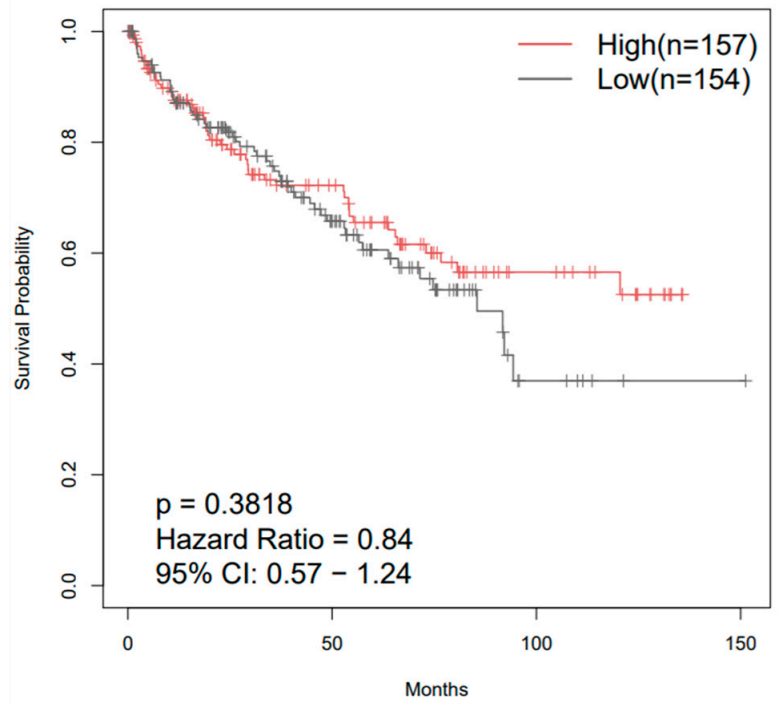

cg09908601

(e)

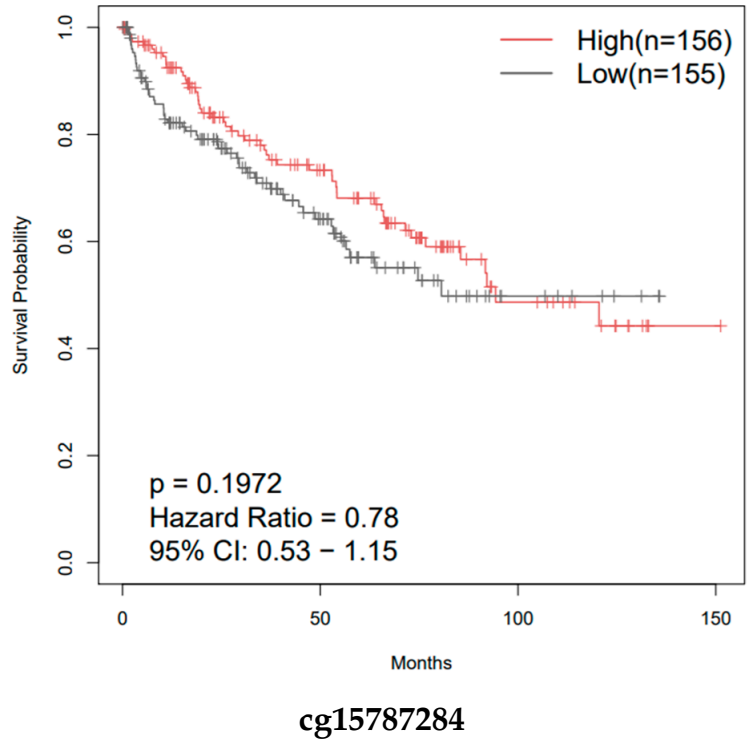

(d)

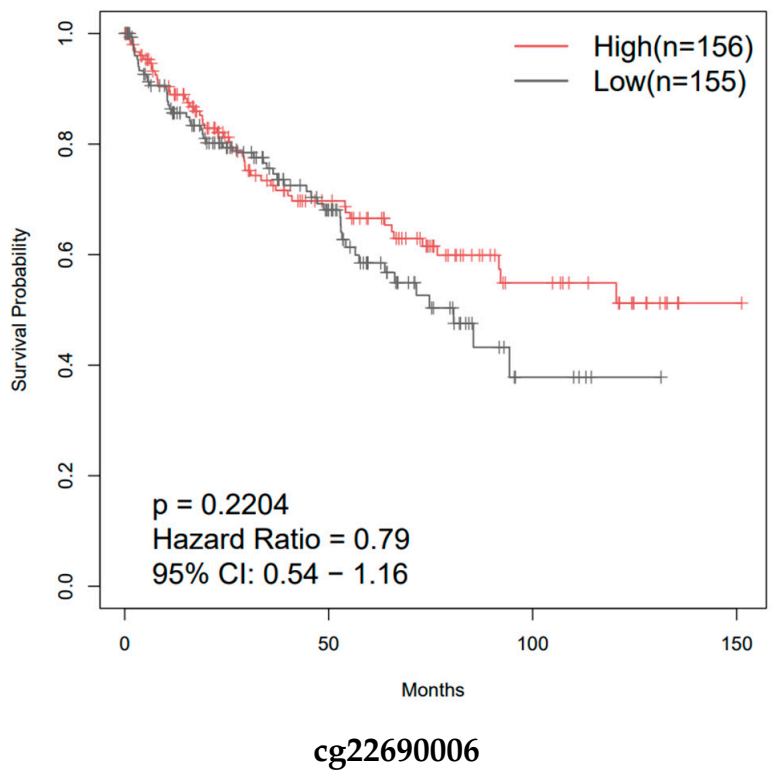

(f)

Figure 4. Cont. 


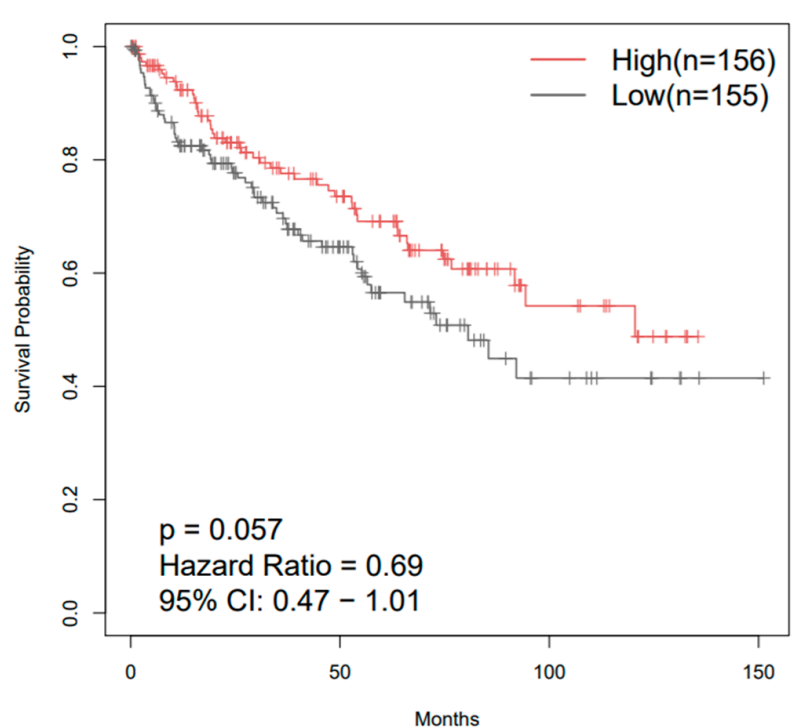

cg18791365

(g)

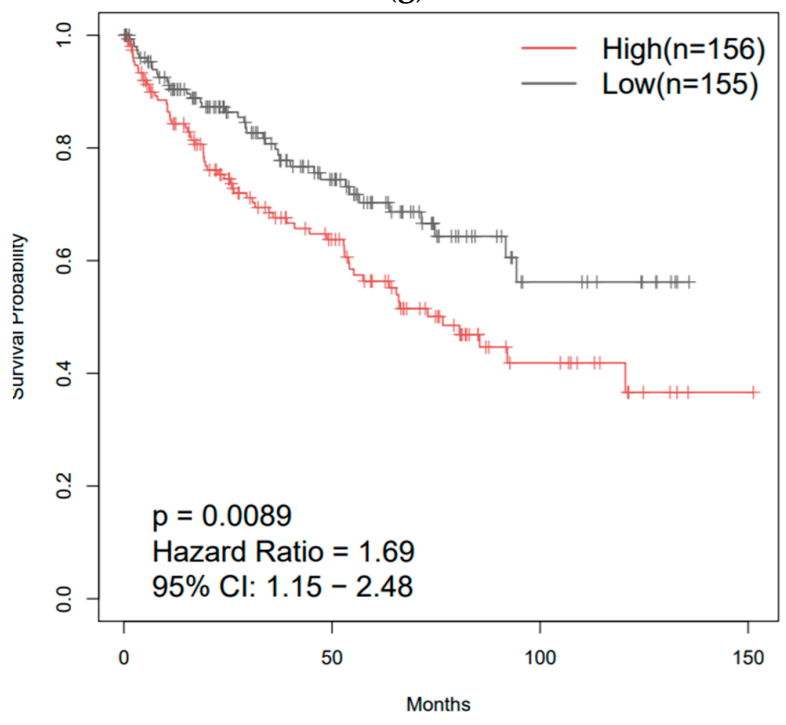

cg01404518

(i)

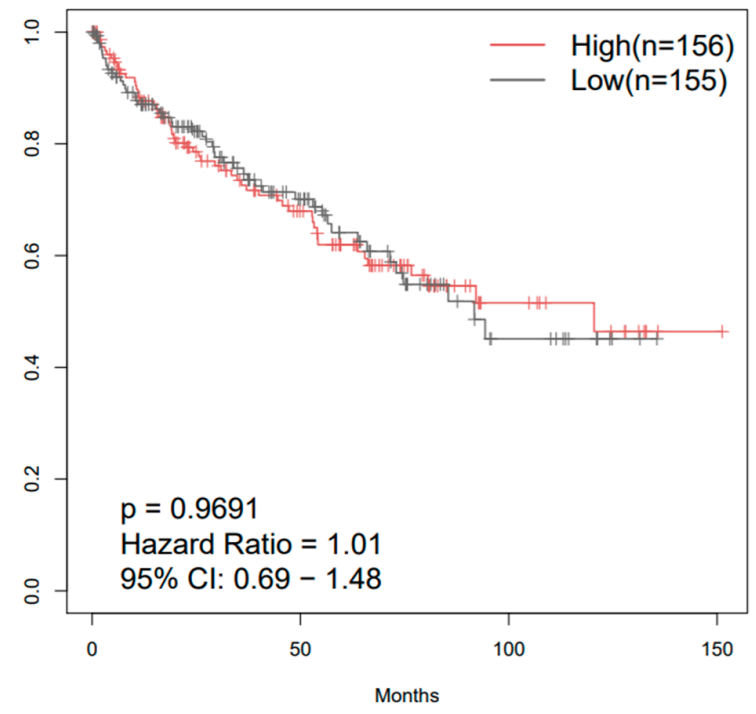

cg03293697

(h)

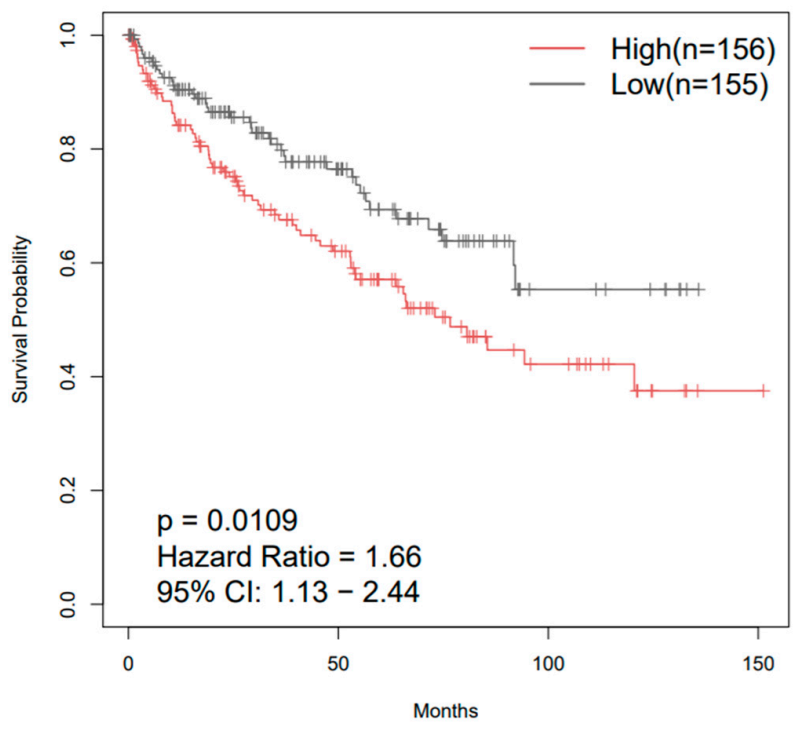

cg18566819

(j)

Figure 4. Methylation status of ZNF433 CpG loci and ZNF433 gene expression in normal and clear-cell renal cell carcinoma (ccRCC) tissues. (a) Methylation analysis between normal and ccRCC tissues revealed that CpG loci associated with ZNF433 were differentially methylated and statistically significant $(p<0.01)$. In addition, analysis of CpG foci in aggregate (last panel) revealed that the ZNF433 gene significantly hypermethylated $\left(p=2.2 \times 10^{-16}\right)$ relative to the normal tissues. (b) Correlational analysis between methylation status at CpG foci and ZNF433 protein expression revealed hypermethylation correlated with protein downregulation $\left(p=2.2 \times 10^{-16}\right)$. (c-j) KM survival plots for CpG islands that displayed differential methylation.

3.5. Effect of BAP1, KDM5C, MTOR, PBRM1, SETD2, and VHL Mutations on ZNF433 $m R N A$ Expression

Since ccRCC is characterized by mutations in proteins that alter epigenetic modifications such as methylation and /or chromatin remodeling, we examined the expression of ccRCC with wildtype and mutated forms of BRCA1 associated protein-1 (BAP1); lysine-specific demethylase 5C (KDMC5); mechanistic target of rapamycin kinase (MTOR); 
polybromo 1 (PBRM1); SET domain containing 2, histone lysine methyltransferase (SETD2); and von Hippel-Lindau tumor suppressor (VHL) genes (Figure 5). ZNF433 expression was significantly depressed in ccRCC with mutations in BAP1 $\left(p=3.3 \times 10^{-11}\right)$, KDM5C $(p=0.042)$, or SETD2 $(p=0.00013)$ genes.

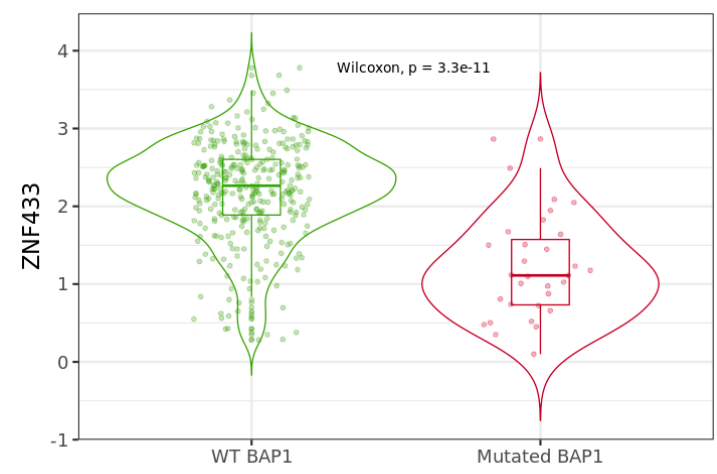

(a)

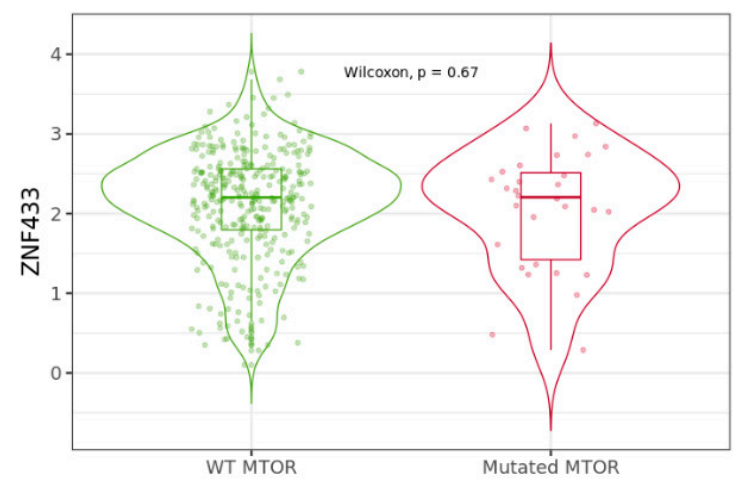

(c)

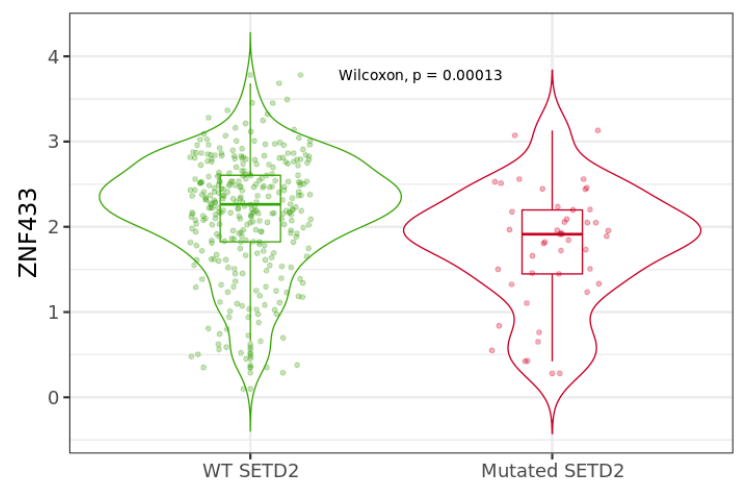

(e)

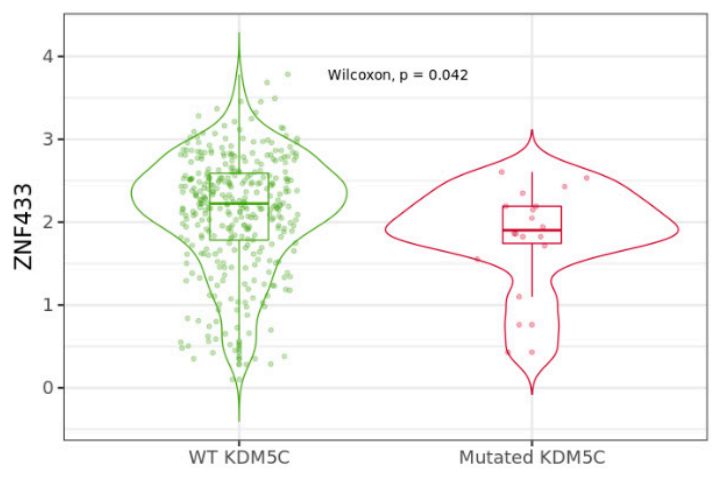

(b)

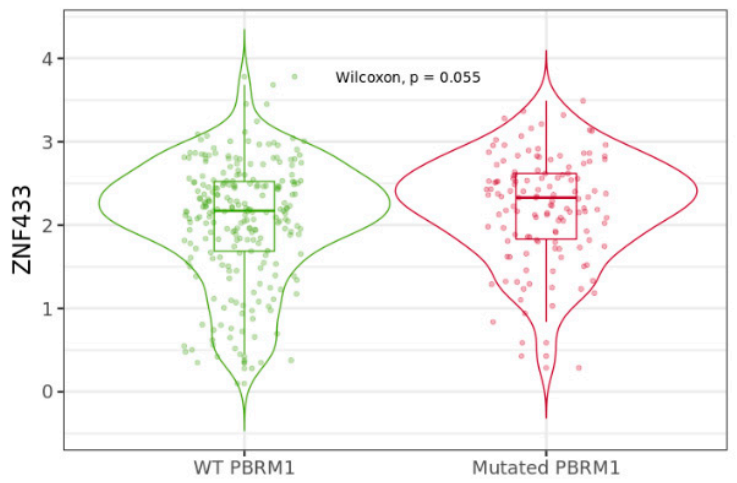

(d)

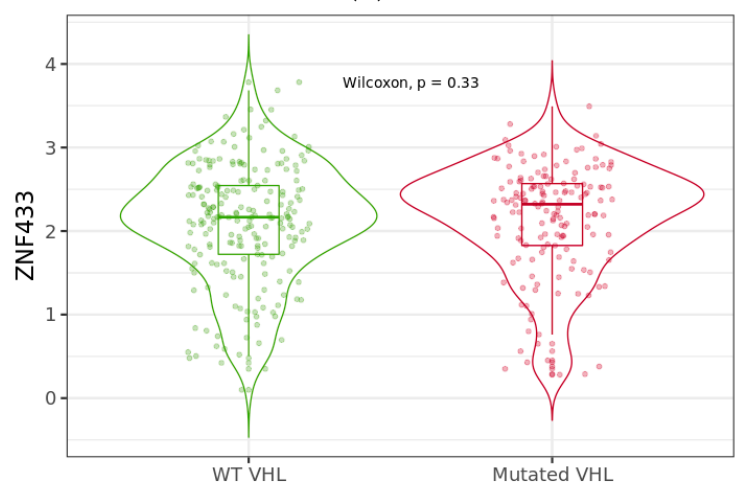

(f)

Figure 5. ZNF433 expression based on mutation status of key tumor-suppressor genes (figures a-f). Violin plots were generated using TIMER2.0 (http:/ / timer.cistrome.org, accessed on 8 May 2021) to analyze ZNF433 expression between mutation status of the most frequently altered genes (a) BAP1, (b) KDM5C, (c) MTOR, (d) PBRM1, (e) SETD2, and (f) VHL that are associated with clear-cell renal cell carcinoma. ZNF433 expression was significantly altered in the presence of BAP1, KDM5C and SETD2. Expression analysis was conducted using RNAseq sample data $(n=367)$ derived from TCGA datasets. Abbreviations: BAP1, BRCA1 associated protein-1; KDMC5, lysine-specific demethylase 5C; mTOR, mechanistic target of rapamycin kinase; PBRM1, polybromo 1; SETD2, SET domain containing 2, histone lysine methyltransferase; VHL, von Hippel-Lindau tumor suppressor. 


\subsection{ZNF433 Pathway Enrichment, Target Gene Expression, and Target Gene Ontology}

Since there is limited information regarding ZNF433 target genes, we examined whether genes correlated highly with ZNF433 expression were components of overrepresented pathways. Specifically, we used the gene set enrichment analysis (GSEA) method to analyze the GO Biological Process and KEGG databases to identify enriched pathways (Figure 6a-k). Figure 6a provides a volcano plot of the negatively (green line) and positively (red lines) correlated genes associated with ZNF433 expression. Figure 6b provides the GSEA analysis of the pathways enriched among genes positively (blue bars) and inversely (orange bars) co-expressed with ZNF433 using the GO Biological Process database. The only pathways that were significantly enriched $(p<0.05$, FDR $<0.05)$ were those connected to the genes inversely co-expressed with ZNF433. Moreover, the enriched pathways were all associated with immune system response/regulation. Figure $6 c-f$ provide the enrichment plots of the top four pathways enriched among the inversely co-expressed genes. These enriched pathways were the adaptive immune response (GO:0002250), neutrophil mediated immunity (GO:0002446), immune response-regulating signaling pathway (GO:0002764), and acute inflammatory response (GO:0002526). The most enriched pathways among the positively correlated genes included those associated with mitochondria/cellular metabolism and protein transport, including GO:0061512: protein localization to cilium (NES $=1.67, \mathrm{FDR}=0.2812, p=0$ ); GO:0098732: macromolecule diacylation (NES $=1.623, \mathrm{FDR}=0.262, p=0.005)$; and GO:0033108: mitochondrial respiratory chain complex assembly (NES $=1.47, \mathrm{FDR}=0.55, p=0.005)$. Similarly, GSEA analysis of the KEGG database revealed that immune-related pathways were predominantly enriched among inversely correlated genes (Figure 6g). Indeed, nine out of the 10 most significantly enriched pathways among inversely correlated genes were associated with defense against infectious organisms (Figure 6g). These included hsa05140 (Leishmaniasis), hsa04145 (Phagosome), hsa05150 (Staphylococcus aureus infection), and hsa05169 (Epstein-Barr infection). Their respective enrichment plots are provided in Figure $6 \mathrm{~h}-\mathrm{k}$. For genes positively co-expressed with ZNF433, GSEA analysis of the KEGG database revealed that most significant pathways associated with cellular metabolism/energy were enriched, and included hsa00280: valine, leucine, and isoleucine degradation $(\mathrm{NES}=1.93, \mathrm{FDR}=0.001$, $p=0)$; hsa00640: propanoate metabolism (NES $=1.86$, FDR $=0.005, p=0)$; and hsa00650: butanoate metabolism (NES $=1.70, \mathrm{FDR}=0.04, p=0$ ). 


\section{ZNF433 Association Result}

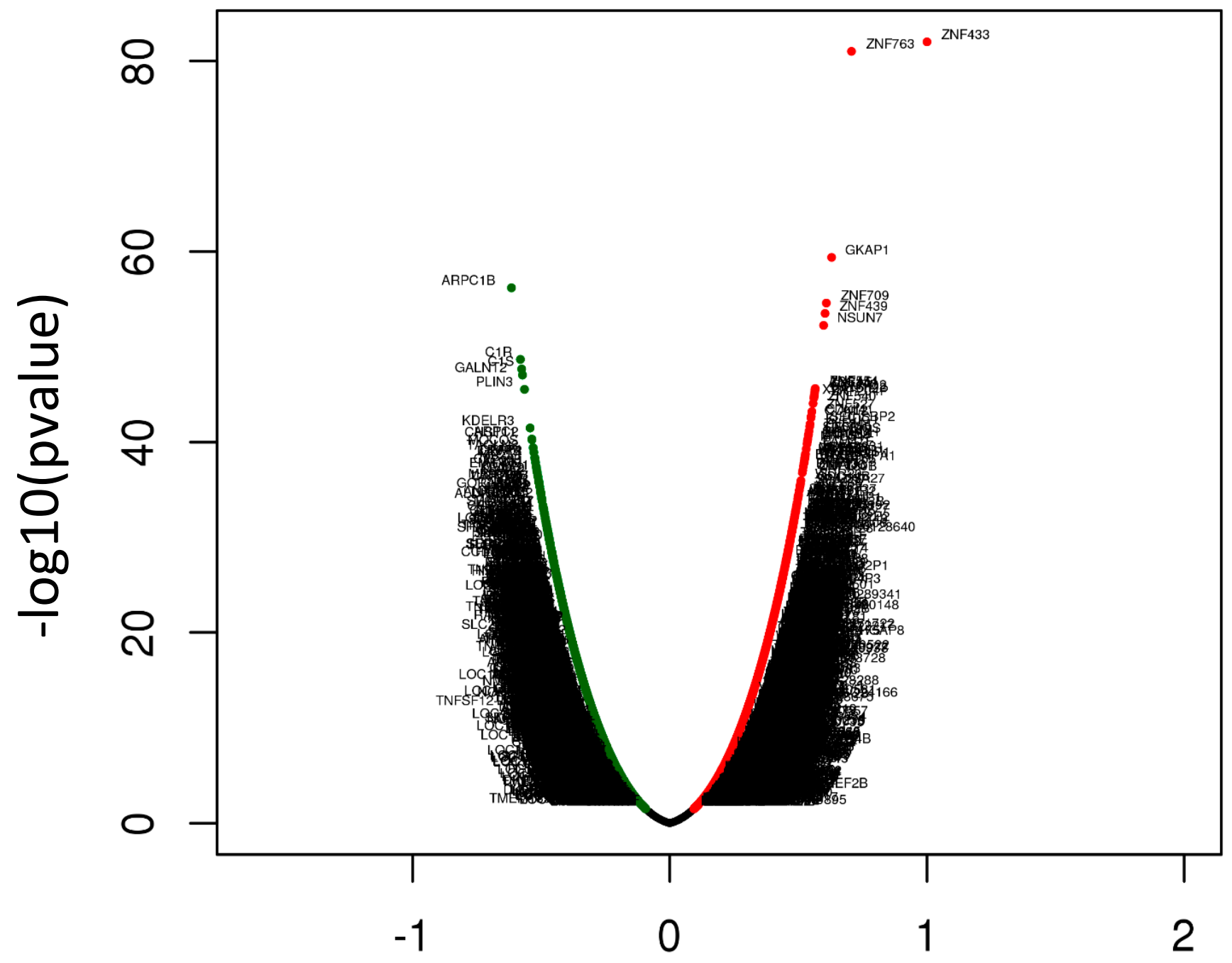

Spearmans rho Statistic (Spearman test)

(a)

Figure 6. Cont. 


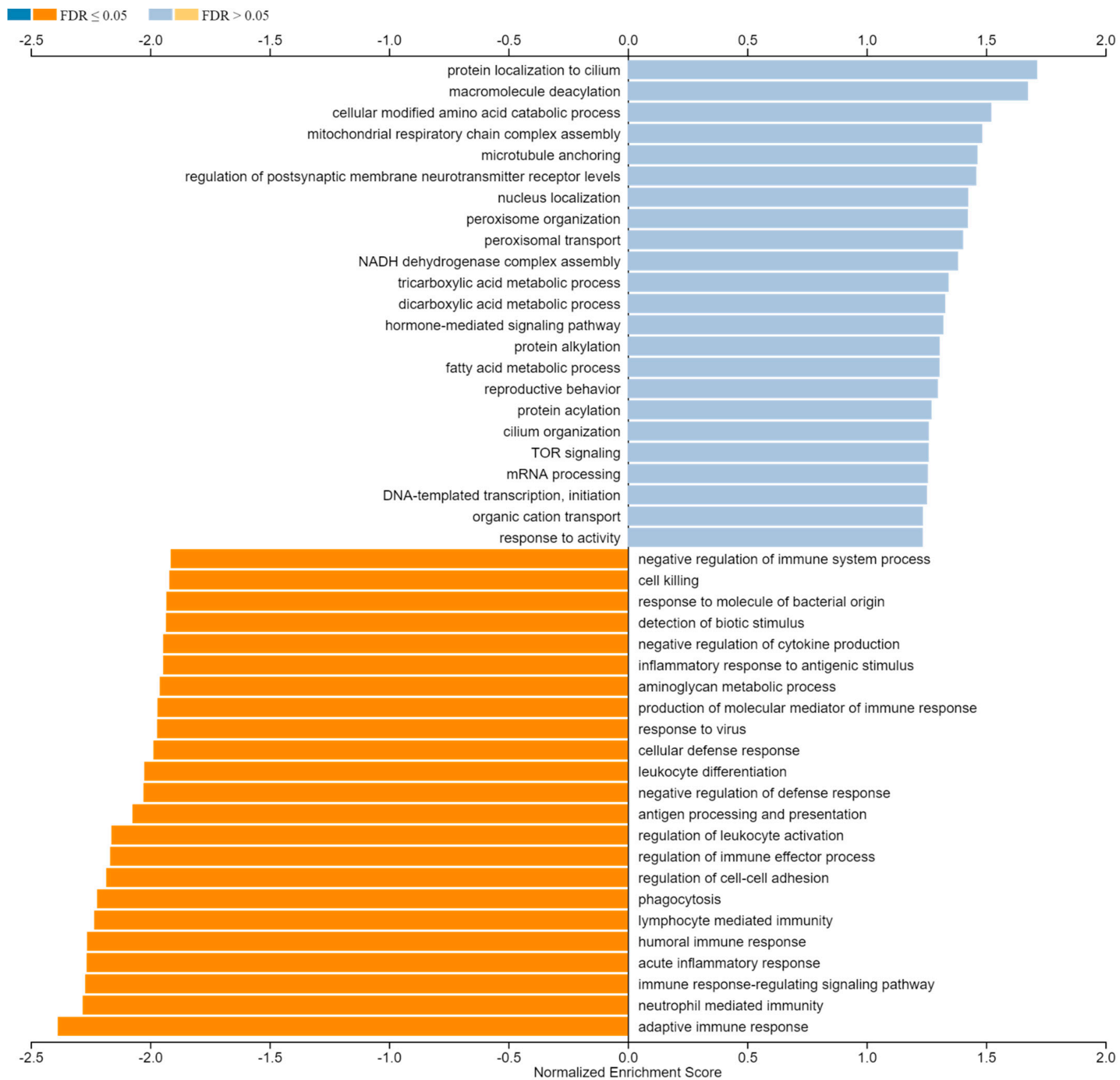

(b)

Figure 6. Cont. 


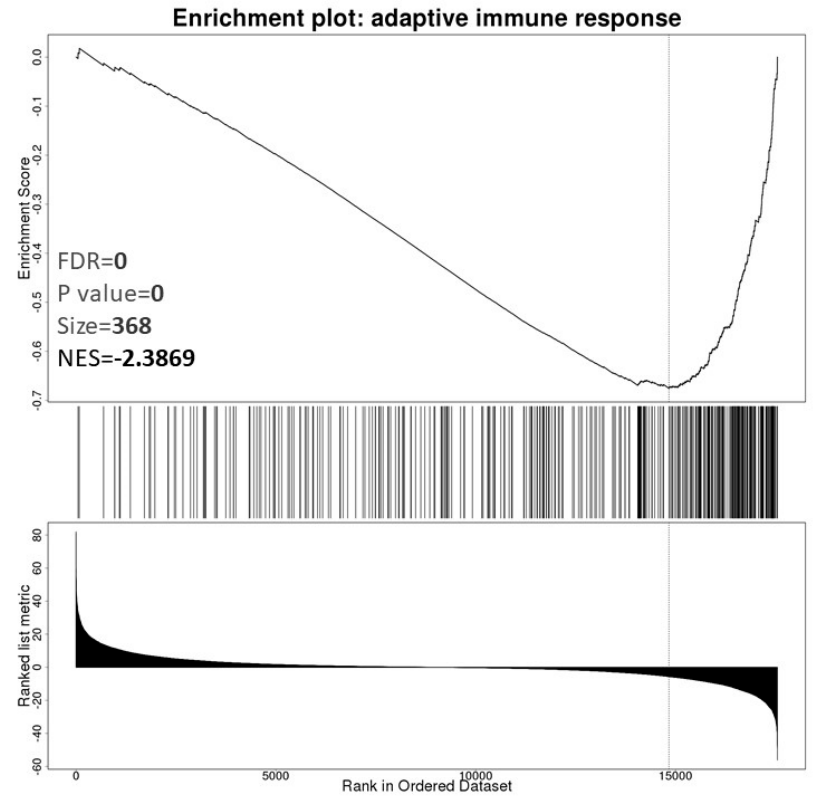

(c)

Enrichment plot: immune response-regulating signaling pathway

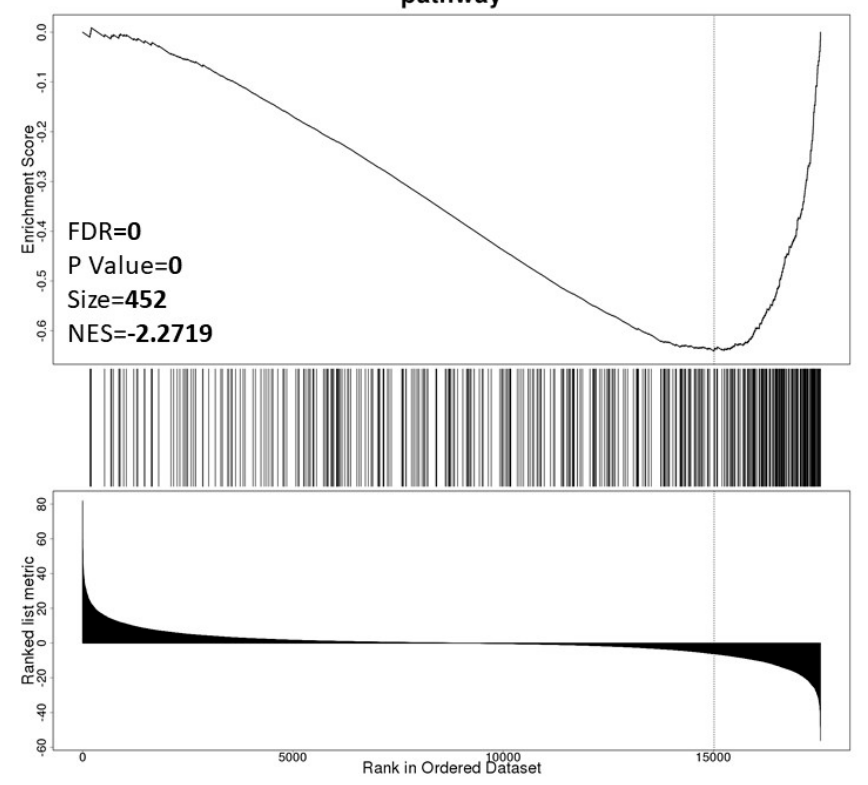

(e)

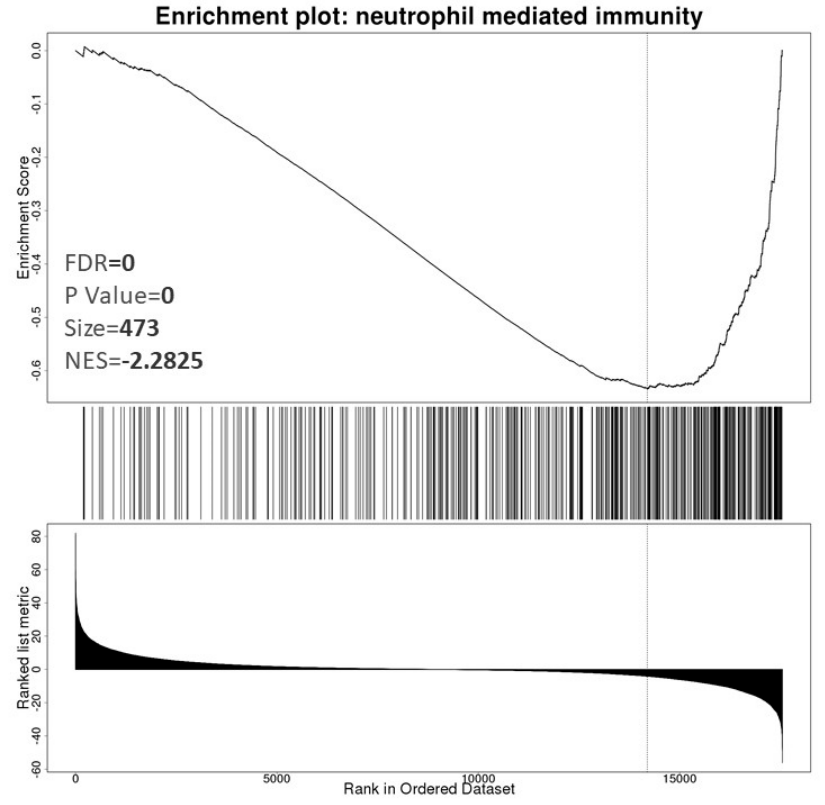

(d)

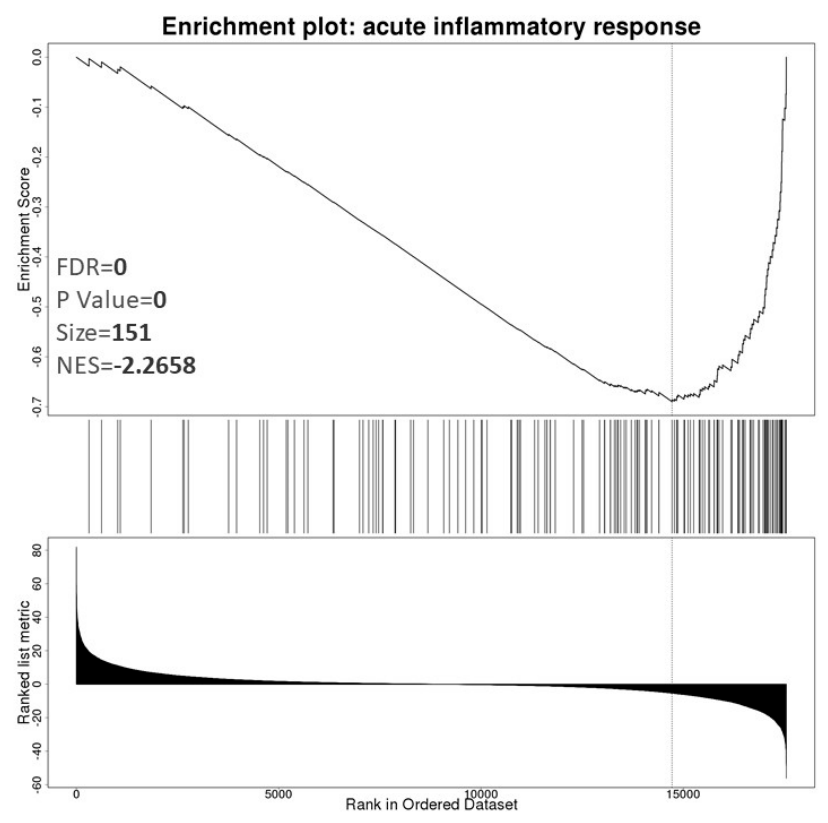

(f)

Figure 6. Cont. 


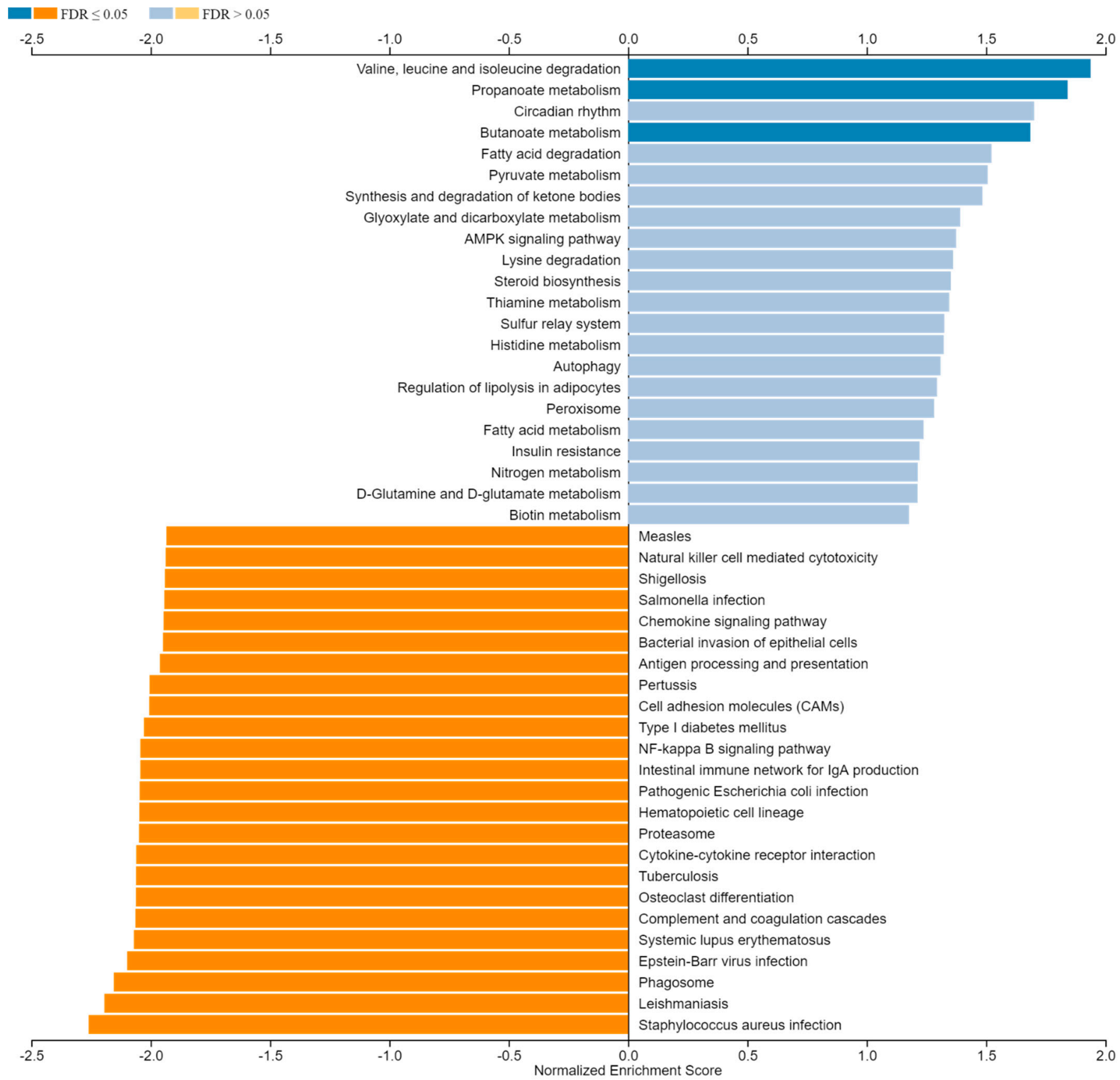

(g)

Figure 6. Cont. 


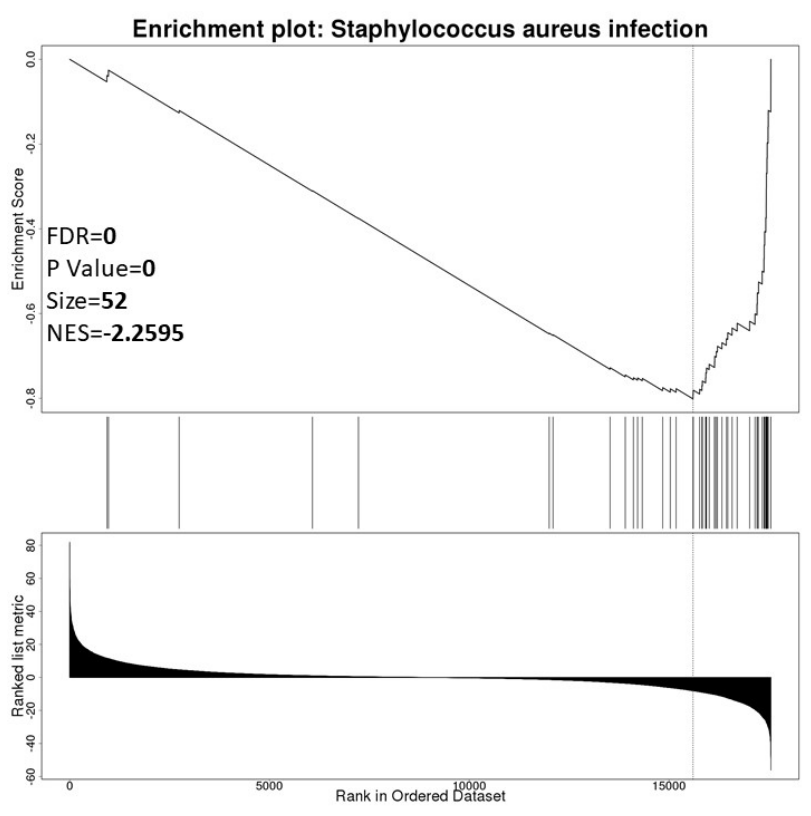

(h)

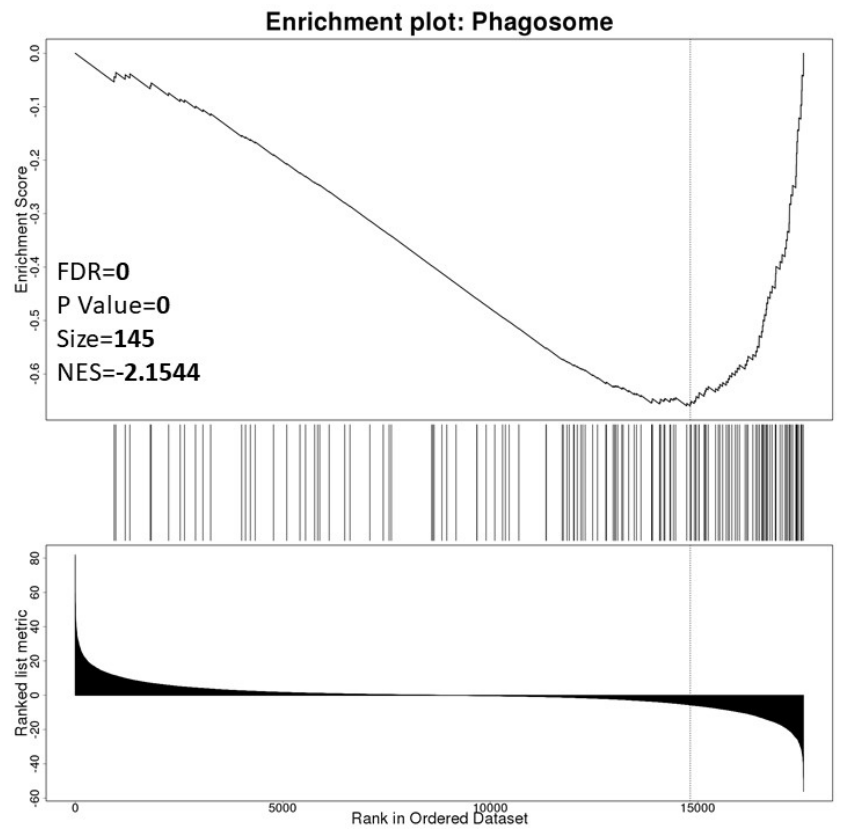

(j)

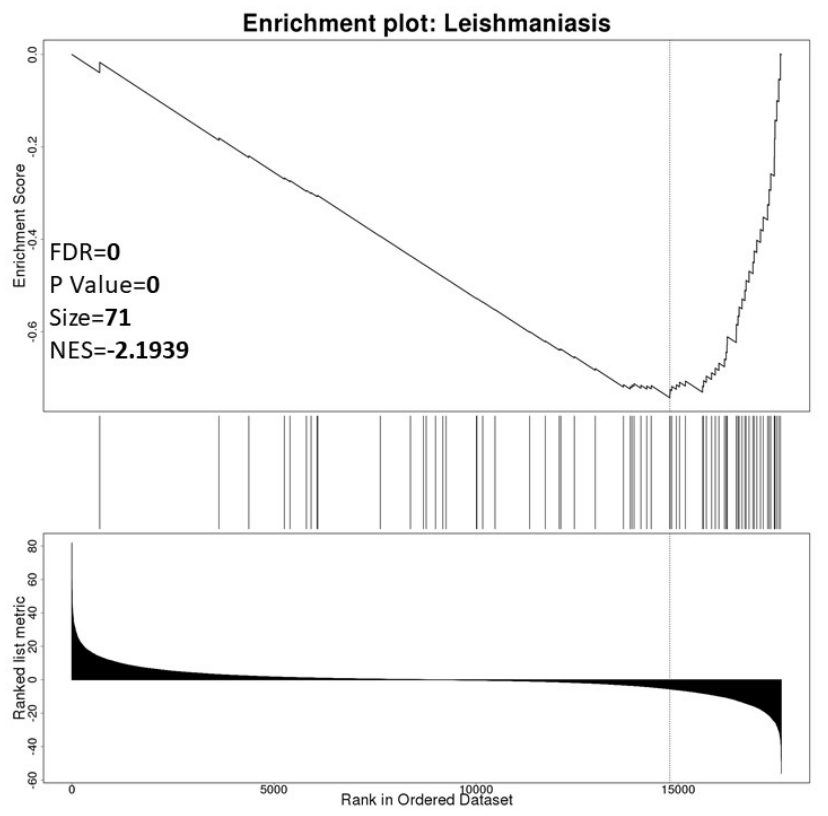

(i)

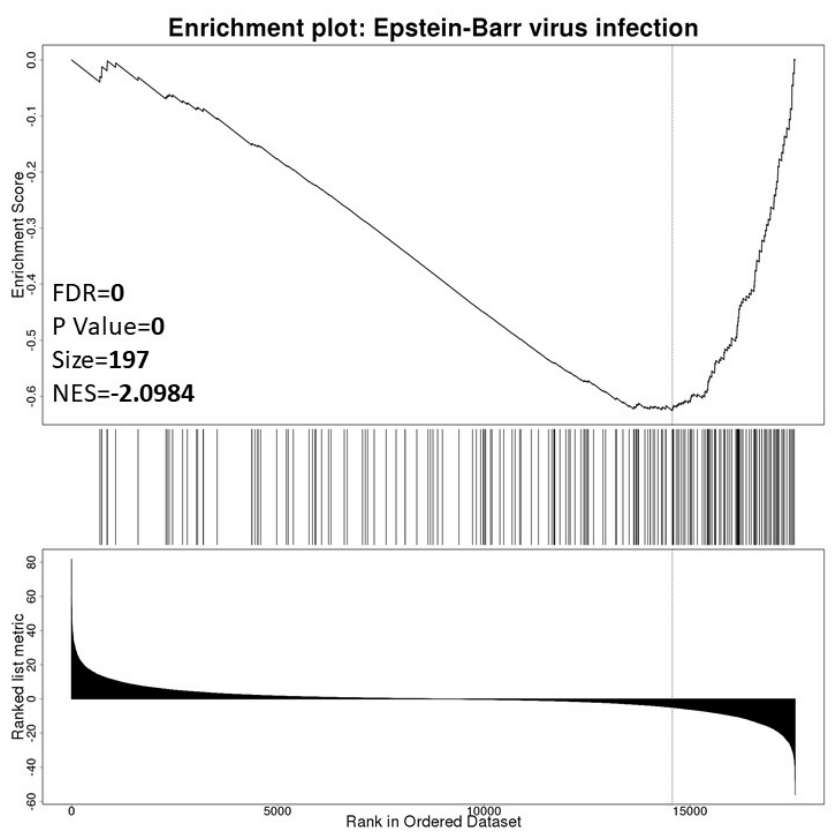

$(\mathbf{k})$

Figure 6. (a) Volcano plots of the negatively and positively correlated genes associated with ZNF433 expression. The green section of the volcano curve represents genes that were inversely correlated with ZNF433 expression, while the red region of the curve represents genes that were positively correlated with ZNF433. (b) GSEA GO Biological Process for genes co-expressed with ZNF433. Blue-colored bars represent pathways enriched in positively correlated genes, with dark blue bars representing FDR $<0.05$, whereas light blue bars are pathways for which FDR $>0.05$. Orange-colored bars represent pathways enriched in inversely correlated genes, with dark orange bars depicting pathways with FDR $<0.05$, and light orange bars representing pathways with FDR $>0.05$. (c-f) Enrichment plots for the top most-significant pathways that were over-represented among inversely correlated genes. (g) GSEA KEGG for genes co-expressed with ZNF433. (h-k) Enrichment plots for the top most-significant pathways over-represented among inversely correlated genes in the KEGG database.

Since ZNF433 belongs to one of the most prominent transcription factor families, we utilized the GSEA Molecular Signature Database to identify 66 putative targets for ZNF433 (Table 2). These putative targets were genes that contained one or more binding sites for UniProt: Q8N7K0 (ZNF433) in their promoter regions (TSS $-1000,+100 \mathrm{bp}$ ), as identified by 
GTRD version 20.06 ChIP-seq harmonization. Figure 7a depicts a heatmap generated from the ZNF433 target gene expression in clear-cell renal cell carcinoma and normal tissues. The most notable targets that showed significant changes in ccRCC relative to normal tissues, and that had been previously implicated in cancer suppression, progression, or tumorigenesis, were COPS3 (fold-change 1.22), RORA (fold-change 1.47), SLC16A1 (MTO1) (fold-change 5.25); RPL36 (fold-change 2.11), SH2B3 (fold-change 2.28), TNFRSF10B (foldchange 2.0); and TNFRSF12A (fold-change 1.54) $(n=535 ; p<0.001)$ (Figure $7 \mathrm{~b}-\mathrm{h}$ ). Even though several ZNF433 target genes were associated with tumorigenesis, gene pathway analysis using the PANTHER web tool revealed no statistically significant pathways enriched within the ZNF433 target gene list. Nonetheless, the largest group of genes were involved in the cellular process (GO:009987) (Figure 7h), and the largest group within the cellular process category was genes involved in cellular metabolic processes (GO: 0044237) (Figure 7i).

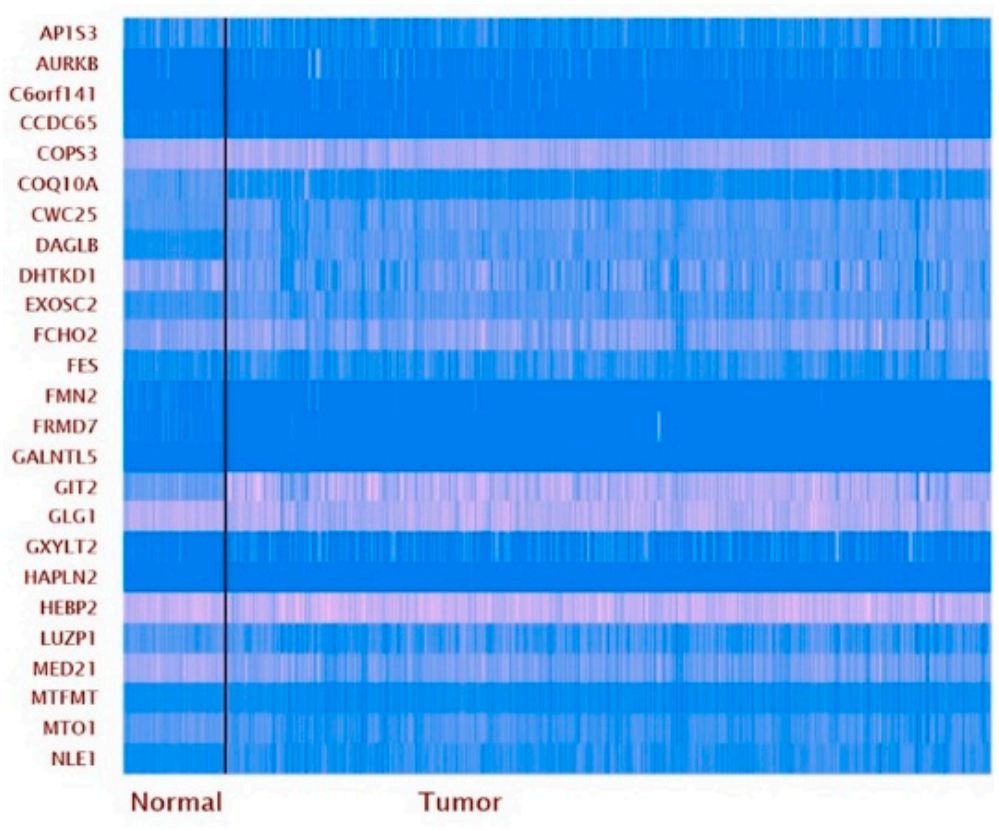

$\log 2(T P M+1)$
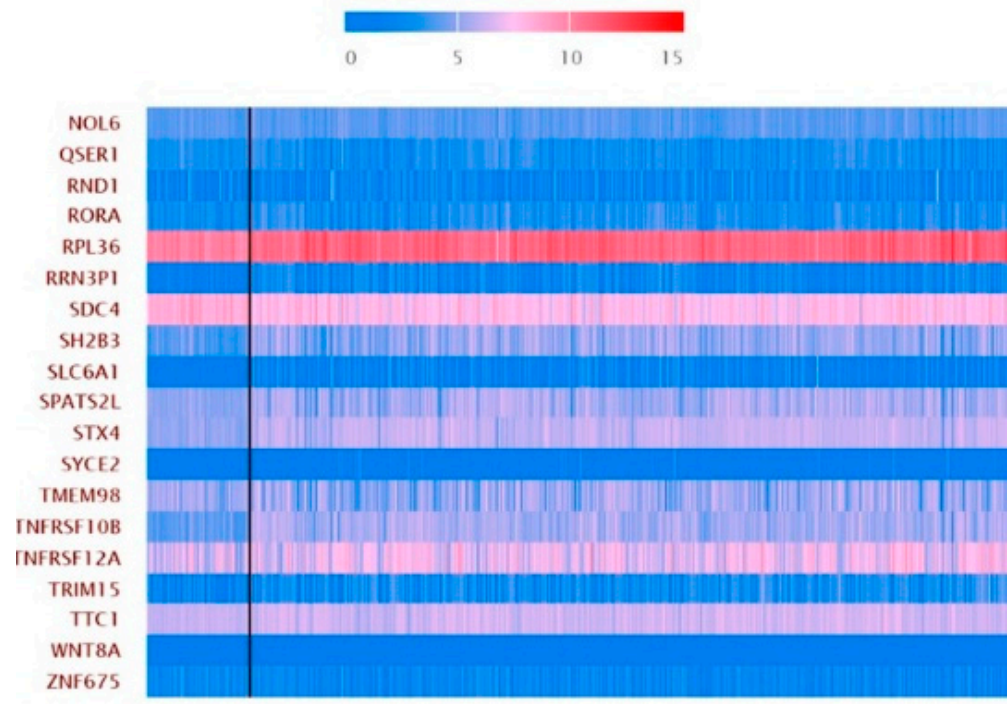

(a)

Figure 7. Cont. 


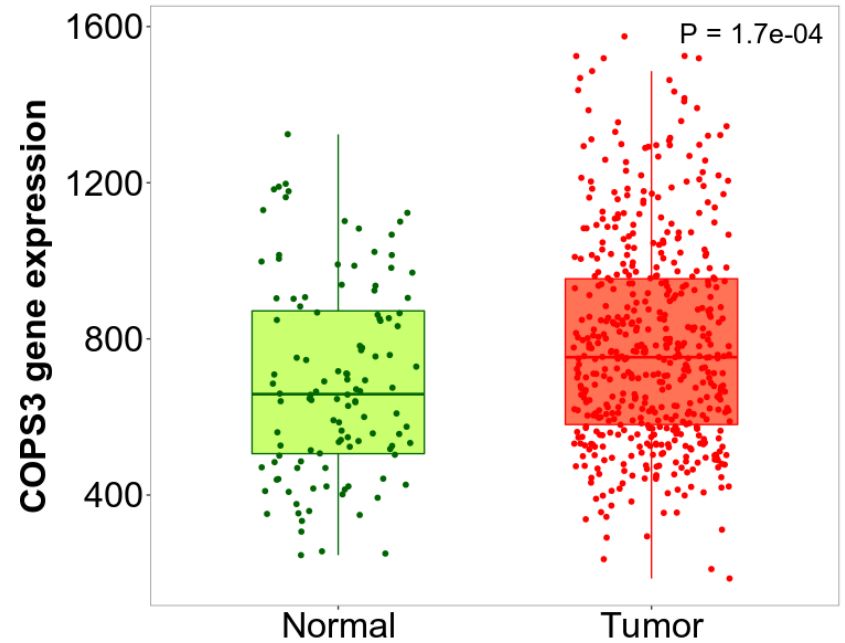

(b)

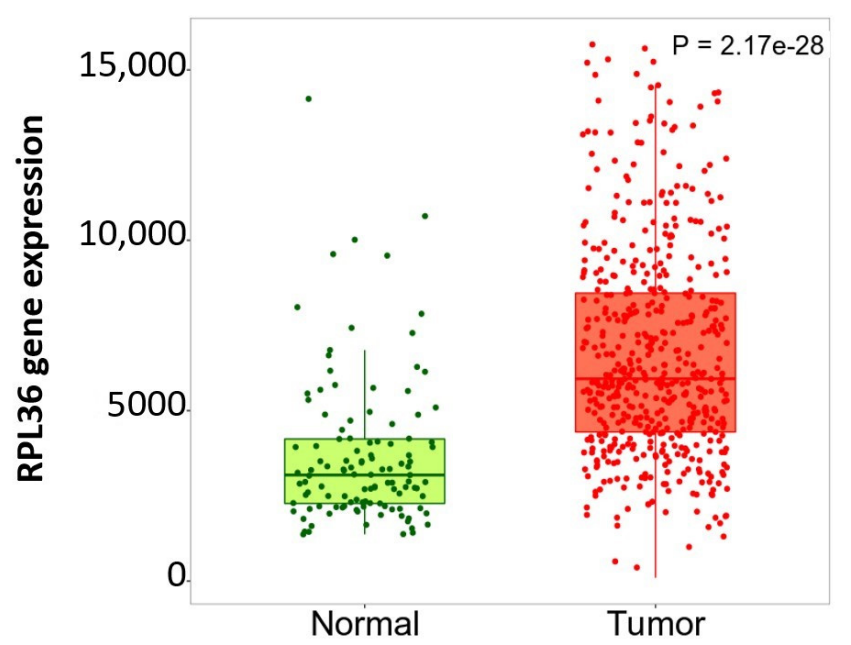

(d)

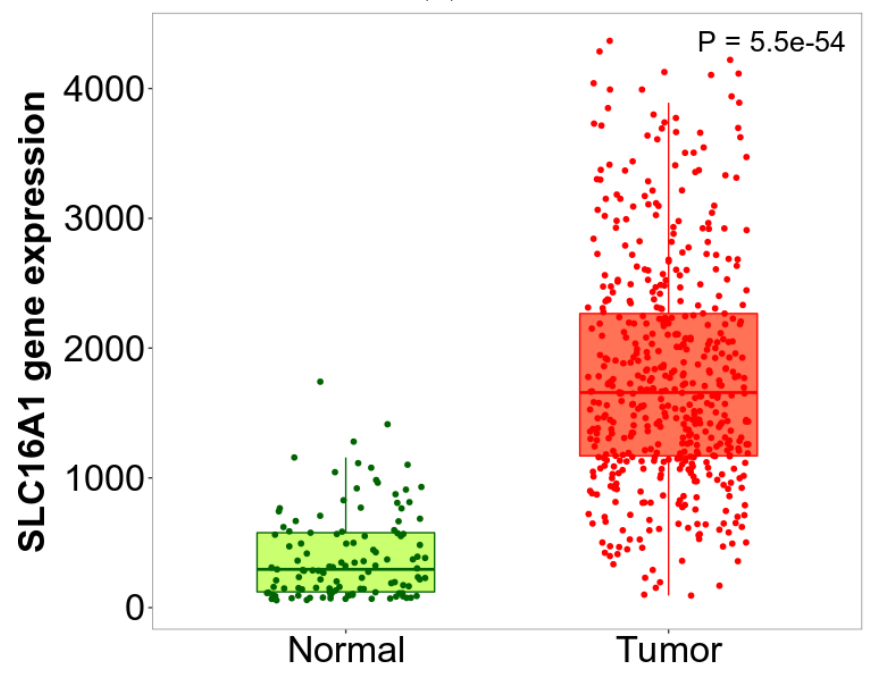

(f)

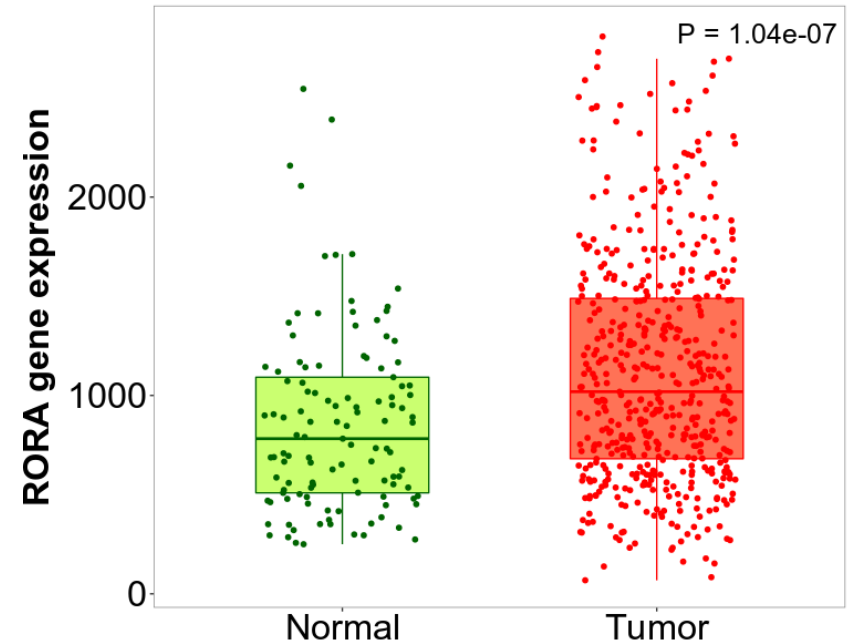

(c)

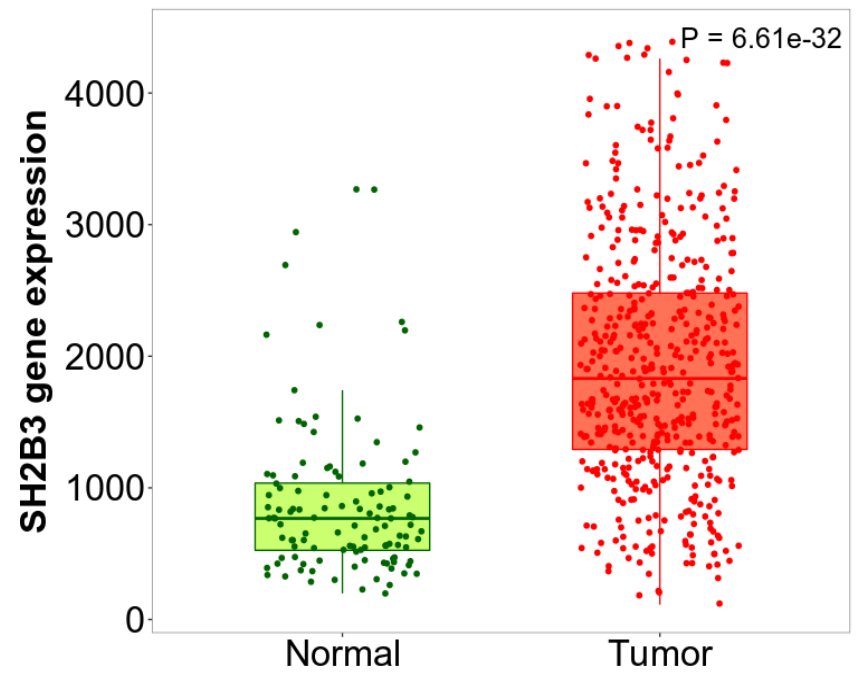

(e)

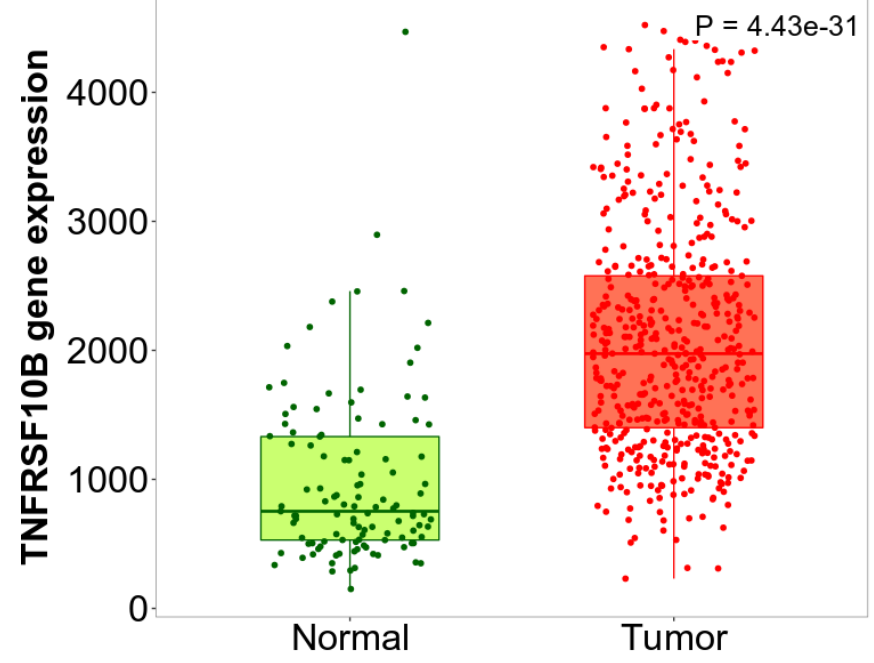

(g)

Figure 7. Cont. 


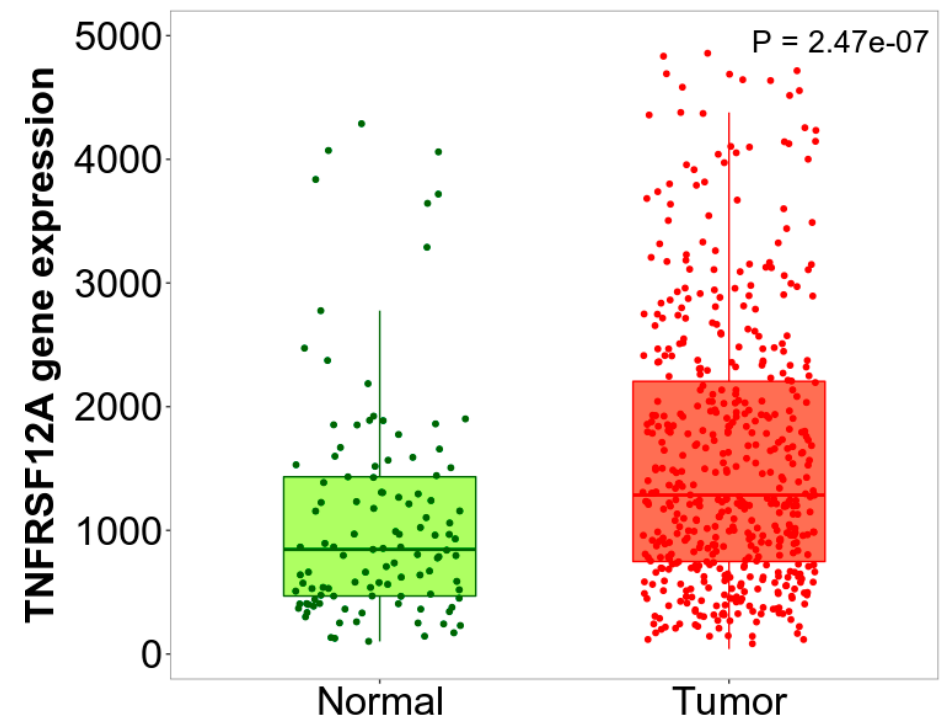

(h)

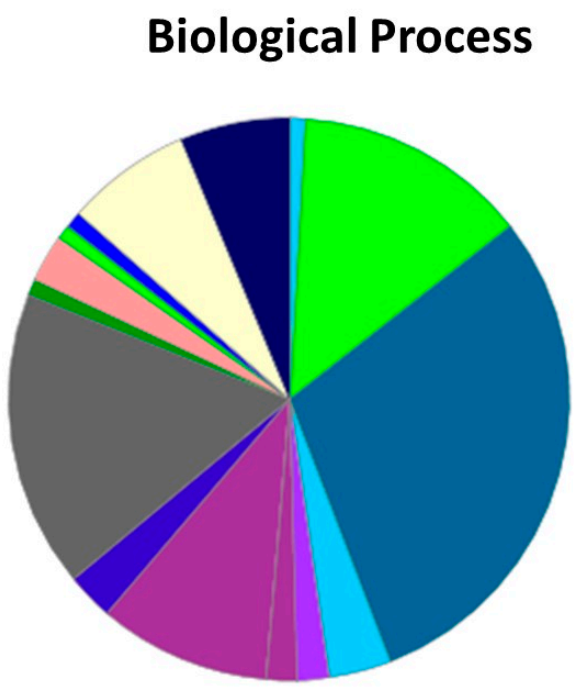

Biological adhesion (GO:0022610), 0.9\%

Biological regulation (GO:0065007), 13.5\%

Cellular process (GO:0009987), 29.7\%

Developmental process (GO:0032502), 3.6\%

$\square$ Immune system process (GO:0002376), 1.8\%

$\square$ Interspecies interaction (GO:0044419), 1.8\%

- Localization (GO:0051179), 9.9\%

- Locomotion (GO:0040011), 2.7\%

Metabolic process (GO:0008152), 17.1\%

Multi-organism process (GO:0051704), 0.9\%

Multicellular organismal process (GO:0032501), 5.8\%

Reproduction (GO:0000003), 0.9\%

- Reproductive process (GO:0022414)), 0.9\%

Response to Stimulus (GO:0050896), 7.2\%

Signaling (GO:0023052)), 6.3\%

(i)

Figure 7. Cont. 


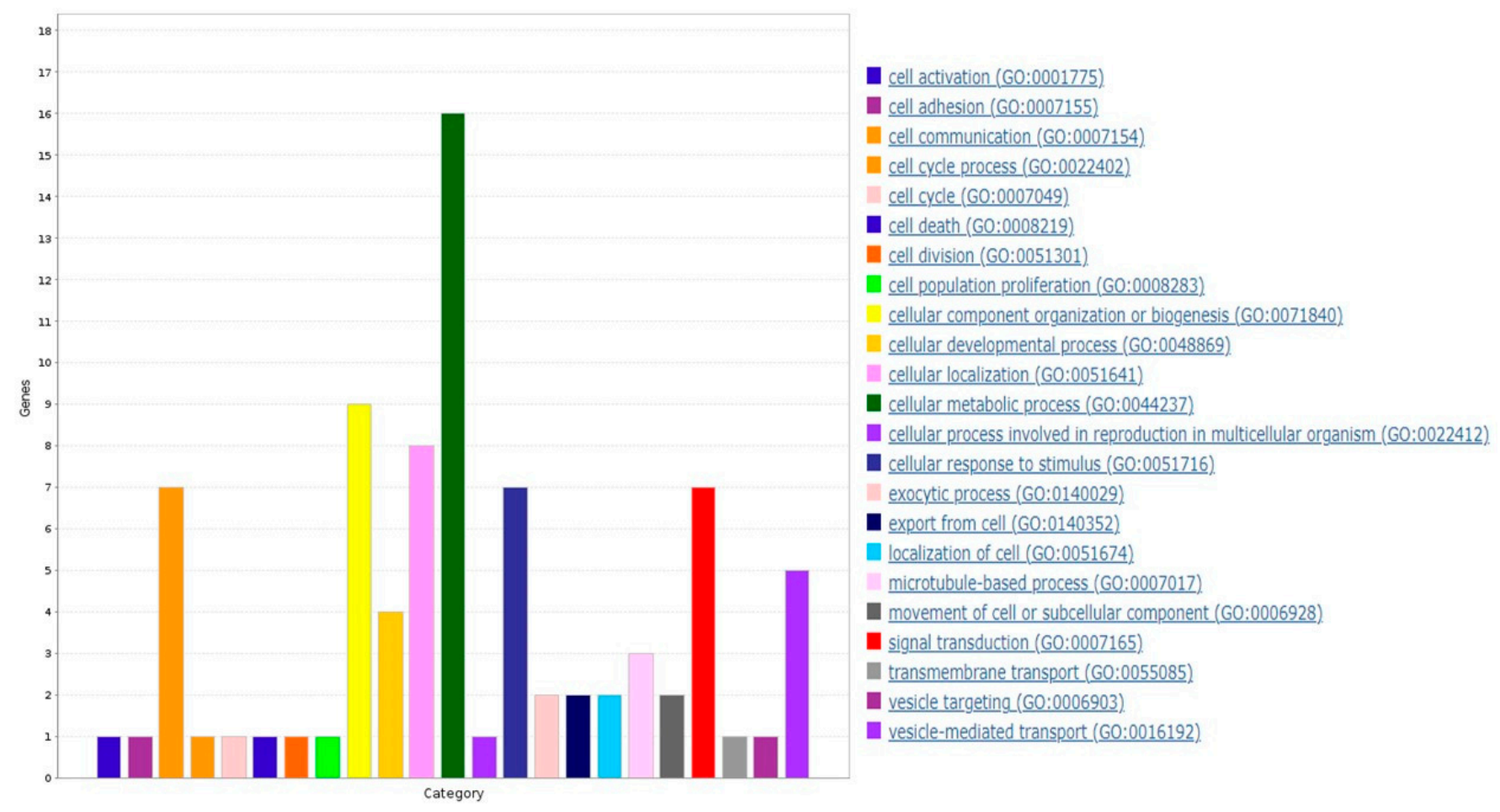

(j)

Figure 7. Expression of potential ZNF433 target genes in clear-cell renal carcinoma. (a) A heatmap generated using UALCAN analysis to determine the expression of predicted ZNF433 target genes in clear-cell renal cell carcinoma. (b-h) Most notable targets implicated in cancer suppression, progression, or tumorigenesis that had significant changes in ccRCC relative to normal, as well as those previously identified $(n=535)$. (i) A pie chart featuring biological processes in which target genes are components, generated using PANTHER (http:/ / pantherdb.org, accessed on 9 May 2021). (j) The largest category of the biological processes was a cellular process (GO: 0009987). The bar graph shows the number of ZNF433 target genes associated with the GO subcategories under the cellular process.

\subsection{Pan-Cancer Analysis of ZNF433 Target Gene Expression}

We examined ZNF433 expression across cancer types (Table 3). ZNF433 was significantly downregulated $(p<0.05)$ in the head and neck, clear-cell renal cell carcinoma, renal papillary, renal chromophobe, lung, and thyroid cancers. Conversely, ZNF433 was significantly upregulated $(p<0.05)$ in bladder, cholangiocarcinoma, liver, and uterine cancers.

Abbreviations used include: ACC, adrenocortical carcinoma; BLCA, bladder urothelial carcinoma; BRCA, breast invasive carcinoma; CESC, cervical squamous cell carcinoma and endocervical adenocarcinoma; $\mathrm{CHOL}$, cholangiocarcinoma; $\mathrm{COAD}$, colon adenocarcinoma; ESCA, esophageal carcinoma; GBM, glioblastoma multiforme; HNSC, head and neck squamous cell carcinoma; $\mathrm{KICH}$, kidney chromophobe; KIRC, kidney renal clear cell carcinoma; KIRP, kidney renal papillary cell carcinoma; LIHC, liver hepatocellular carcinoma; LUAD, lung adenocarcinoma; LUSC, lung squamous cell carcinoma; PAAD, pancreatic adenocarcinoma; PCPG, pheochromocytoma and paraganglioma; PRAD, prostate adenocarcinoma; READ, rectum adenocarcinoma; SKCM, skin cutaneous melanoma; STAD, stomach adenocarcinoma; THCA, thyroid carcinoma; UCEC, uterine corpus endometrial carcinoma. 
Table 3. Expression profile of ZNF433 in different cancers. Statistical significance was set at $p<0.05$.

\begin{tabular}{|c|c|c|c|}
\hline Tumor & Normal & Change & $\begin{array}{c}\text { Significance } \\
(p-\text { Value })\end{array}$ \\
\hline BLCA.Tumor $(n=408)$ & BLCA.Normal $(n=19)$ & Upregulation & 0.016096887 \\
\hline BRCA.Tumor $(n=1093)$ & BRCA.Normal $(n=112)$ & Downregulation & 0.446186328 \\
\hline CESC.Tumor $(n=304)$ & CESC.Normal $(n=3)$ & Downregulation & 0.210693518 \\
\hline CHOL.Tumor $(n=36)$ & CHOL.Normal $(n=9)$ & Upregulation & 0.005768157 \\
\hline COAD.Tumor $(n=457)$ & COAD.Normal $(n=41)$ & Upregulation & 0.792680029 \\
\hline ESCA.Tumor $(n=184)$ & ESCA.Normal $(n=11)$ & Downregulation & 0.164913994 \\
\hline GBM.Tumor $(n=153)$ & GBM.Normal $(n=5)$ & Upregulation & 0.001122476 \\
\hline HNSC.Tumor $(n=520)$ & HNSC.Normal $(n=44)$ & Downregulation & $6.12 \times 10^{-8}$ \\
\hline KICH.Tumor $(n=66)$ & KICH.Normal $(n=25)$ & Downregulation & $8.04 \times 10^{-5}$ \\
\hline KIRC.Tumor $(n=533)$ & KIRC.Normal $(n=72)$ & Downregulation & $1.13 \times 10^{-23}$ \\
\hline KIRP.Tumor $(n=290)$ & KIRP.Normal $(n=32)$ & Downregulation & $2.86 \times 10^{-9}$ \\
\hline LIHC.Tumor $(n=371)$ & LIHC.Normal $(n=50)$ & Upregulation & $7.93 \times 10^{-11}$ \\
\hline LUAD.Tumor $(n=515)$ & LUAD.Normal $(n=59)$ & Downregulation & 0.039291777 \\
\hline LUSC.Tumor $(n=501)$ & LUSC.Normal $(n=51)$ & Downregulation & $1.16 \times 10^{-5}$ \\
\hline PAAD.Tumor $(n=178)$ & PAAD.Normal $(n=4)$ & Downregulation & 0.112226334 \\
\hline PCPG.Tumor $(n=179)$ & PCPG.Normal $(n=3)$ & Upregulation & 0.032947137 \\
\hline PRAD.Tumor $(n=497)$ & PRAD.Normal $(n=52)$ & Upregulation & 0.028391067 \\
\hline READ.Tumor $(n=166)$ & READ.Normal $(n=10)$ & Downregulation & 0.101167394 \\
\hline SKCM.Tumor $(n=103)$ & SKCM.Metast $(n=368)$ & Downregulation & 0.826588455 \\
\hline STAD.Tumor $(n=415)$ & STAD.Normal $(n=35)$ & Downregulation & 0.433244729 \\
\hline THCA.Tumor $(n=501)$ & THCA.Normal $(n=59)$ & Downregulation & $1.98 \times 10^{-11}$ \\
\hline UCEC.Tumor $(n=545)$ & UCEC.Normal $(n=35)$ & Upregulation & 0.015728415 \\
\hline
\end{tabular}

\section{Discussion}

Though highly treatable if the diagnosis is early, clear-cell renal cell carcinoma (ccRCC) is the most common and aggressive form of urological cancers [31]. Unfortunately, 20-30\% of renal carcinomas are detected in later stages and are often associated with treatment challenges and poorer prognosis. Thus, there is a need to identify prognostic markers which can be used to detect ccRCC. Zinc-finger proteins are the largest family of transcription factors. Moreover, given that cancers are characterized by abnormal gene transcription, these proteins represent a significant opportunity to identify molecular signatures, which could serve as either prognostic or predictive factors for neoplastic diseases, including clear-cell renal cell carcinoma. Indeed, Krüppel-associated box domain zinc-finger proteins (KRAB-ZFPs) are often dysregulated in cancer cells [8]. For instance, uterine corpus endometrial carcinoma, colon and rectal adenocarcinomas, and skin cutaneous melanoma possess significant mutations in the Krüppel-associated box (KRAB) repressor domains in the Cys2His2 subfamily of zinc-finger proteins [32]. KRAB-ZNFs such as ZNF695, ZNF468, ZNF714, ZNF320, ZNF273, ZNF525, ZNF530, ZNF643, ZNF138, ZNF92, ZNF200, ZNF707, ZNF205, ZNF485, ZNF354A, and ZNF789 are differentially expressed across multiple cancer cohorts $[8,11]$. Moreover, recent studies suggest that KRAB-ZNFs can modulate tumorigenesis. For example, ZNF217 overexpression is associated with poorly differentiated tumors and the progression of ovarian cancers [33]. Restoration of KRAB-ZNF382 expression in silenced ESCC cells suppresses tumor-cell proliferation and metastasis by inducing cell apoptosis [34]. Overexpression of ZNF671 inhibited EMT, migration, and invasion of CNS cancers, lung cancer, melanoma, and breast carcinoma in vitro [18]. Taken collectively, KRAB-ZNF proteins play a significant role in tumorigenesis, and altered expression may serve as biomarkers, oncogenes, or tumor-suppressor genes. 
In this study, data indicated that ZNF433 behaved in a consistent manner, with it being a prognostic marker and/or a putative tumor-suppressor gene. ZNF433 belongs to the Krüppel-associated box (KRAB) $\mathrm{C} 2 \mathrm{H} 2$-type zinc-finger protein subfamily, and like many other members of this subfamily, is part of a cluster of KRAB-ZNFs found on chromosome 19 q13 [35]. Because of the sheer number of ZNF genes located in this region, the functions of these proteins and their roles in tumorigenesis have not been fully elucidated. ZNF433 gene and protein expression are reduced in clear-cell renal cell carcinoma (Figure 1a,b). Expression is stage- and grade-specific, with mRNA levels being significantly suppressed in higher stages (III and IV) and grades (III and IV) compared to lower grades and stages (I and II) (Figure 2a-d). These data strongly suggest that ZNF433 may mediate tumorigenesis, and loss of function is associated with cancer progression and aggressiveness. Indeed, transcript expression decreased progressively with metastatic nodal involvement (Figure 2c) and was significantly lower (Figure 2d) in the more aggressive, higher-risk ccB renal carcinoma subtype [36]. In addition, lower transcription expression was associated with low patient overall survival (Figure 3a), thereby suggesting that ZNF433 may play a protective role against tumorigenesis in normal kidney tissues.

Since ZNF433 levels are significantly reduced in ccRCC, we investigated whether gene suppression could be due to epigenetic mechanisms. The ZNF433 promoter and CpG foci were found to be significantly hypermethylated. Moreover, hypermethylation was negatively correlated with mRNA expression, thereby suggesting that ZNF433 suppression may be due, in part, to aberrant methylation. Because ccRCC is also characterized by mutations in genes that are involved in chromatin remodeling and epigenetic regulations such as BAP1, KDM5C, MTOR, PBRM1, SETD2, and VHL [37-42], we examined the effects of the most commonly altered genes on ZNF433 expression. ZNF433 transcripts were significantly reduced in BAP1, SETD2, and KDM5C mutants, with BAP1 mutants exerting the greatest suppressive effects. The BAP1 gene encodes a ubiquitin carboxyl-terminal hydrolase, a deubiquitinase enzyme that regulates a number of processes through modification of histones and regulating chromatin scaffolding. BAP1 functions as a major tumor suppressor that mediates DNA damage repair, cell-cycle control, chromatin modification, programmed cell death, and the immune response [43]. Indeed, loss function in the BAP1 gene is associated with several aggressive cancers, including clear-cell renal cell carcinomas [43]. SETD2 encodes a methyltransferase that specifically trimethylates lysine-36 of histone H3 (H3K36me3), whereas KDM5c (also known as JARID1C) is a gene that encodes an $\mathrm{H} 3 \mathrm{~K} 4 \mathrm{me} 2 / 3$ demethylase that plays a central role in transcriptional repression and can mediate cancer progression [44]. These three genes are all involved in epigenetic controls and chromatin modeling, suggesting that histone modification, like DNA methylation, is essential to transcriptional control of ZNF433.

Given that ZNF433 is a putative transcription factor, we identified potential target genes using the GSEA Molecular Signature Database (Table 4). We examined the expression of ZNF433 target genes in clear-cell renal cell carcinoma. Several target genes were differentially expressed, and associated with cancer suppression, progression, or tumorigenesis (Figure $6 \mathrm{a}, \mathrm{b}$ ). The most notable target genes (Figure $7 \mathrm{~b}-\mathrm{h}$ ) were potential oncogenes/promoters of tumorigenesis (COPS3, RORA, SLC16A1), and potential tumor suppressors (RPL36, SH2B3, TNFRSF10B, TNFRSF12A) [45-48]. In addition, we used PANTHER to annotate ZNF433 target genes (Figure $7 \mathrm{~h}, \mathrm{i}$ ). The largest portion of genes $(29.7 \%)$ were associated with biological processes (GO:0009987) (Figure 7i), and within the category of biological processes, the largest number of genes were involved in cellular metabolic processes (GO:0044237) (Figure 7h,i). However, given the small numbers of possible target genes, no pathway or process was statistically over-represented. Nonetheless, it should be noted that clear-cell renal cell carcinoma is especially characterized by impaired cellular metabolism and metabolic reprogramming characterized by increased glycolysis and lactate production, and abnormal accumulations of lipids and reduced oxidative phosphorylation $[49,50]$. 
Table 4. List of predicted ZNF433 target genes identified using the GSEA Molecular Signature Database. These are genes containing one or more binding sites for UniProt: Q8N7K0 (ZNF433) in their promoter regions (TSS $-1000,+100$ bp) as identified by GTRD version 20.06 ChIP-seq harmonization.

\begin{tabular}{|c|c|}
\hline Gene Symbol & Official Name \\
\hline ACER3 & alkaline ceramidase 3 \\
\hline ALDOA & aldolase, fructose-bisphosphate A \\
\hline ANGPTL6 & angiopoietin-like 6 \\
\hline AP1S3 & adaptor related protein complex 1 subunit sigma 3 \\
\hline ASS1P5 & argininosuccinate synthetase 1 pseudogene 5 \\
\hline AURKB & aurora kinase B \\
\hline BORCS6 & BLOC-1 related complex subunit 6 \\
\hline C6orf141 & chromosome 6 open reading frame 141 \\
\hline CCDC65 & coiled-coil domain containing 65 \\
\hline COPS3 & COP9 signalosome subunit 3 \\
\hline COQ10A & coenzyme Q10A \\
\hline CROCCP3 & CROCC pseudogene 3 \\
\hline CWC25 & CWC25 spliceosome associated protein homolog \\
\hline CYP1B1-AS1 & CYP1B1 antisense RNA 1 \\
\hline DAGLB & diacylglycerol lipase beta \\
\hline DHTKD1 & dehydrogenase E1 and transketolase domain containing 1 \\
\hline EXOSC2 & exosome component 2 \\
\hline FABP5P3 & fatty-acid-binding protein 5 pseudogene 3 \\
\hline FCHO2 & FCH and mu domain-containing endocytic adaptor 2 \\
\hline FES & FES proto-oncogene, tyrosine kinase \\
\hline FMN2 & formin 2 \\
\hline FRMD7 & FERM domain containing 7 \\
\hline GALNTL5 & polypeptide $\mathrm{N}$-acetylgalactosaminyltransferase like 5 \\
\hline GIT2 & GIT ArfGAP 2 \\
\hline GLG1 & Golgi glycoprotein 1 \\
\hline GPR1-AS & GPR1 antisense RNA \\
\hline GXYLT2 & glucoside xylosyltransferase 2 \\
\hline HAPLN2 & hyaluronan and proteoglycan link protein 2 \\
\hline HEBP2 & heme binding protein 2 \\
\hline KAT8 & lysine acetyltransferase 8 \\
\hline LINC01235 & long intergenic non-protein coding RNA 1235 \\
\hline LINC01641 & long intergenic non-protein coding RNA 1641 \\
\hline LUZP1 & leucine zipper protein 1 \\
\hline MED21 & mediator complex subunit 21 \\
\hline MTFMT & mitochondrial methionyl-tRNA formyl transferase \\
\hline MTND1P14 & MT-ND1 pseudogene 14 \\
\hline MTO1 & mitochondrial tRNA translation optimization 1 \\
\hline NLE1 & notchless homolog 1 \\
\hline NOL6 & nucleolar protein 6 \\
\hline NUCB1-AS1 & NUCB1 antisense RNA 1 \\
\hline OR1X5P & olfactory receptor family 1 subfamily $X$ member 5 pseudogene \\
\hline QSER1 & glutamine and serine rich 1 \\
\hline RN7SL93P & RNA, 7SL, cytoplasmic 93, pseudogene \\
\hline RND1 & Rho family GTPase 1 \\
\hline RNU6-1003P & RNA, U6 small nuclear 1003, pseudogene \\
\hline RNU6-166P & RNA, U6 small nuclear 166, pseudogene \\
\hline RORA & RAR related orphan receptor $A$ \\
\hline RPL32P27 & ribosomal protein L32 pseudogene 27 \\
\hline RPL36 & ribosomal protein L36 \\
\hline RRN3P1 & RRN3 pseudogene 1 \\
\hline SDC4 & syndecan 4 \\
\hline SH2B3 & SH2B adaptor protein 3 \\
\hline SLC6A1 & solute carrier family 6 -member 1 \\
\hline SNHG30 & small nucleolar RNA host gene 30 \\
\hline
\end{tabular}


Table 4. Cont.

\begin{tabular}{ll}
\hline \multicolumn{1}{c}{ Gene Symbol } & \multicolumn{1}{c}{ Official Name } \\
\hline SPATS2L & spermatogenesis associated serine rich 2 like \\
STX4 & syntaxin 4 \\
SYCE2 & synaptonemal complex central element protein 2 \\
TMEM98 & transmembrane protein 98 \\
TNFRSF10B & TNF receptor superfamily member 10b \\
TNFRSF12A & TNF receptor superfamily member 12A \\
TRAV15 & T cell receptor alpha variable 15 (pseudogene) \\
TRIM15 & tripartite motif containing 15 \\
TTC1 & tetratricopeptide repeat domain 1 \\
TTLL13P & tubulin tyrosine ligase like 13, pseudogene \\
WNT8A & Wnt family member 8A \\
ZNF675 & zinc-finger protein 675 \\
\hline
\end{tabular}

ZNF433 may be involved in cellular metabolism. Indeed, pathway analysis of the KEGG database suggested that genes positively co-expressed with ZNF433 are associated with metabolism. Though not rising to the level of significance as defined by $p$-values and FDR less than 0.05, positively correlated genes in the GO Biological Processes database were mainly associated with cellular energetics and mitochondrial function, further suggesting that ZNF433 may be involved in the regulation of cellular energetics. It should be noted that altered cellular energetics may impact the ccRCC tumor microenvironment by regulating angiogenesis and inflammatory signatures [51]. Clear-cell renal cell carcinoma is highly infiltrated with T cells, and therapeutic interventions such as the use of MTOR inhibitors and checkpoint inhibitors (PD-1 and PD-L1) have been shown to be effective in the management of ccRCC [52]. However, recent studies have shown that $\mathrm{T}$ cells may become dysfunctional or "exhausted" due to aberrant mitochondrial functions and cellular energetics [52-55]. Interestingly, GSEA analysis of the Gene Ontology Biological Processes database, as well as the KEGG database, revealed that pathways that were enriched in immune function were over-represented in genes negatively co-expressed with ZNF433. In the case of GO Biological Processes, all enriched pathways associated with inverse expression of ZNF433 were related to immune function. Hence, ZNF433 may be intimately involved in the dysregulation of cellular energetic and immune function.

Lastly, we examined ZNF433 expression across multiple cancers to ascertain if ZNF433 may be a common tumor marker (Table 3). ZNF433 expression was upregulated $(p<0.05)$ in bladder urothelial carcinoma, cholangiocarcinoma, glioblastoma multiforme, liver hepatocellular carcinoma, pheochromocytoma and paraganglioma, prostate adenocarcinoma, and uterine corpus endometrial carcinoma; while expression was suppressed $(p<0.05)$ in head and neck squamous cell carcinoma, kidney chromophobe, kidney renal clear-cell carcinoma, kidney renal papillary cell carcinoma, lung adenocarcinoma, lung squamous cell carcinoma, and thyroid carcinoma, thereby indicating that ZNF433 expression is altered across multiple tumor cohorts and thus may play a general role in cancer formation and progression.

\section{Conclusions}

In conclusion, ZNF433 is a member of the KRAB-ZFTF subfamily, which is differentially expressed in several cancers; however, poor prognosis is largely associated with low expression in kidney cancers. Although its role in renal carcinoma is yet to be fully defined, it is clear that this gene suppression is associated with higher cancer stages, tumor grades, degree of metastasis, and more aggressive subtypes of ccRCC. Therefore, ZNF433 represents a predictive risk factor for tumor progression, and may prove to be a possible therapeutic target in the treatment of ccRCC. Though additional large-scale validation is warranted, we anticipate that assessing ZNF433 mRNA expression in biopsies will be most beneficial as a diagnostic tool for screening and identifying patients at higher risks of developing the aggressive ccRCC subtypes. Given the scarcity of information regarding 
the transcriptional targets of ZNF433, comprehensive analysis is required to identify and validate ZNF433 gene targets, as well as transcription regulators of ZNF433, particularly in clear-cell renal cell carcinomas.

Author Contributions: Conceptualization, S.O.H., M.D.S. and R.R.R.; methodology, S.O.H.; resources, K.F.A.S.; writing—original draft preparation, S.O.H.; writing—review and editing, K.F.A.S., R.R.R. and M.D.S.; funding acquisition, K.F.A.S. All authors have read and agreed to the published version of the manuscript.

Funding: This research was supported by the National Institute of Minority Health and Health Disparities of the National Institutes of Health through grant number U54 MD 007582.

Institutional Review Board Statement: Not applicable.

Informed Consent Statement: Not applicable.

Data Availability Statement: Datasets utilized to analyze the clinicopathological features of ZNF433 and target genes can be assessed with the UALCAN webtool (http:/ / ualcan.path.uab.edu/analysis. html, accessed on 5 May 2021). Data collection for the UALCAN analysis webtool has been previously described in detail [18]. Specifically, for clear-cell renal cell carcinoma, the KIRC-TCGA was downloaded from the TCGA database. To investigate ZNF433 and its putative target associated with mRNA expression, tumor stage, grade, and subtype, 533 tumor samples and 72 normal tissues were taken for analysis. Due to the lack of cancer stage information, two samples were excluded, leaving 531 tumor samples for further analysis. For analysis of gene expression relative to tumor grade, eight samples were excluded due to inefficient information regarding histological grade, leaving 525 tumor samples. For ZNF433 expression in metastatic tissues, 277 samples were excluded because cancer in nearby lymph nodes (Nx) could not be measured, leaving 256 tumor samples for analysis. Lastly, 153 samples were excluded for analysis of ccRCC subtypes because no information regarding subtypes was available, leaving 380 tumor samples.

Conflicts of Interest: The funders had no role in the design of the study; in the collection, analyses, or interpretation of data; in the writing of the manuscript; or in the decision to publish the results.

\section{References}

1. Eble, J.N.; Sauter, G.; Epstein, J.I.; Sesterhenn, I.A. Renal Cell Carcinoma. In World Health Organization Classification of Tumours. Pathology and Genetics of Tumours of the Urinary System and Male Genital Organs; IARC Press: Lyon, France, 2004 ; pp. 8-14.

2. Dimitrieva, S.; Schlapbach, R.; Rehrauer, H. Prognostic value of cross-omics screening for kidney clear cell renal cancer survival. Biol. Direct. 2016, 11, 68. [CrossRef]

3. Low, G.; Huang, G.; Fu, W.; Moloo, Z.; Girgis, S. Review of renal cell carcinoma and its common subtypes in radiology. World J. Radiol. 2016, 8, 484-500. [CrossRef]

4. Powles, T.; Albiges, L.; Staehler, M.; Bensalah, K.; Dabestani, S.; Giles, R.H.; Hofmann, F.; Hora, M.; Kuczyk, M.A.; Lam, T.B.; et al. Updated European association of urology guidelines recommendations for the treatment of first-line metastatic clear cell renal cancer. Eur. Urol. 2017, 73, 311-315. [CrossRef]

5. Huang, M.; Chen, Y.; Han, D.; Lei, Z.; Chu, X. Role of the zinc finger and SCAN domain-containing transcription factors in cancer. Am. J. Cancer Res. 2019, 9, 816-836.

6. Krishna, S.S.; Majumdar, I.; Grishin, N.V. Structural classification of zinc fingers: Survey and summary. Nucleic Acids Res. 2003, 31, 532-550. [CrossRef]

7. Urrutia, R. KRAB-containing zinc-finger repressor proteins. Genome Biol. 2003, 4, 231. [CrossRef]

8. Sobocińska, J.; Molenda, S.; Machnik, M.; Oleksiewicz, U. KRAB-ZFP Transcriptional Regulators Acting as Oncogenes and Tumor Suppressors: An Overview. Int. J. Mol. Sci. 2021, 22, 2212. [CrossRef]

9. Wuttke, D.S.; Foster, M.P.; Case, D.A.; Gottesfeld, J.M.; Wright, P.E. Solution structure of the first three zinc fingers of TFIIIA bound to the cognate DNA sequence: Determinants of affinity and sequence specificity. J. Mol. Biol. 1997, 273, 183-206. [CrossRef]

10. Lupo, A.; Cesaro, E.; Montano, G.; Zurlo, D.; Izzo, P.; Costanzo, P. KRAB-zinc finger proteins: A repressor family displaying multiple biological functions. Curr. Genom. 2013, 14, 268-278. [CrossRef]

11. Machnik, M.; Cylwa, R.; Kiełczewski, K.; Biecek, P.; Liloglou, T.; Mackiewicz, A.; Oleksiewicz, U. The expression signature of cancer-associated KRAB-ZNF factors identified in TCGA pan-cancer transcriptomic data. Mol. Oncol. 2019, 13, 701-724. [CrossRef]

12. Sun, R.; Xiang, T.; Tang, J.; Peng, W.; Luo, J.; Li, L.; Qiu, Z.; Tan, Y.; Ye, L.; Zhang, M.; et al. 19q13 KRAB zinc-finger protein ZNF471 activates MAPK10/JNK3 signaling but is frequently silenced by promoter CpG methylation in esophageal cancer. Theranostics 2020, 10, 2243-2259. [CrossRef] 
13. Tao, Y.F.; Hu, S.Y.; Lu, J.; Cao, L.; Zhao, W.L.; Xiao, P.F.; Xu, L.X.; Li, Z.H.; Wang, N.N.; Du, X.J.; et al. Zinc finger protein 382 is downregulated by promoter hypermethylation in pediatric acute myeloid leukemia patients. Int. J. Mol. Med. 2014, 34, $1505-1515$. [CrossRef]

14. Pei, L.; He, X.; Li, S.; Sun, R.; Xiang, Q.; Ren, G.; Xiang, T. KRAB zinc-finger protein 382 regulates epithelial-mesenchymal transition and functions as a tumor suppressor but is silenced by $\mathrm{CpG}$ methylation in gastric cancer. Int. J. Oncol. 2018, 53, 961-972. [CrossRef]

15. Tao, C.; Luo, J.; Tang, J.; Zhou, D.; Feng, S.; Qiu, Z.; Putti, T.C.; Xiang, T.; Tao, Q.; Li, L.; et al. The tumor suppressor Zinc finger protein 471 suppresses breast cancer growth and metastasis through inhibiting AKT and Wnt/ $\beta$-catenin signaling. Clin. Epigenetics 2020, 12, 173. [CrossRef]

16. Zhang, J.; Wen, X.; Liu, N.; Li, Y.Q.; Tang, X.R.; Wang, Y.Q.; He, Q.M.; Yang, X.J.; Zhang, P.P.; Ma, J.; et al. Epigenetic mediated zinc finger protein 671 downregulation promotes cell proliferation and tumorigenicity in nasopharyngeal carcinoma by inhibiting cell cycle arrest. J. Exp. Clin. Cancer Res. 2017, 36, 147. [CrossRef]

17. Zhang, J.; Luo, J.; Jiang, H.; Xie, T.; Zheng, J.; Tian, Y.; Li, R.; Wang, B.; Lin, J.; Xu, A.; et al. The Tumor Suppressor Role of Zinc Finger Protein 671 (ZNF671) in Multiple Tumors Based on Cancer Single-Cell Sequencing. Front. Oncol. 2019, 9, 1214. [CrossRef]

18. Chandrashekar, D.S.; Bashel, B.; Balasubramanya, S.A.H.; Creighton, C.J.; Ponce-Rodriguez, I.; Chakravarthi, B.V.; Varambally, S. UALCAN: A Portal for Facilitating Tumor Subgroup Gene Expression and Survival Analyses. Neoplasia 2017, 19, 649-658. [CrossRef]

19. Bartha, Á.; Balázs, G. TNMplot.com: A Web Tool for the Comparison of Gene Expression in Normal, Tumor and Metastatic Tissues. Int. J. Mol. Sci. 2021, 22, 2622. [CrossRef]

20. Li, T.; Fan, J.; Wang, B.; Traugh, N.; Chen, Q.; Liu, J.S.; Li, B.; Liu, X.S. TIMER: A web server for comprehensive analysis of tumor-infiltrating immune cells. Cancer Res. 2017, 77, e108-e110. [CrossRef]

21. Li, T.; Fu, J.; Zeng, Z.; Cohen, D.; Li, J.; Chen, Q.; Li, B.; Liu, X.S. TIMER 2.0 for analysis of tumor-infiltrating immune cells. Nucleic Acids Res. 2020, J48, W509-W514. [CrossRef]

22. Pontén, F.; Jirström, K.; Uhlen, M. The Human Protein Atlas-A tool for pathology. J. Pathol. 2008, 216, 387-393. [CrossRef]

23. Györffy, B.; Lanczky, A.; Eklund, A.C.; Denkert, C.; Budczies, J.; Li, Q.; Szallasi, Z. An online survival analysis tool to rapidly assess the effect of 22,277 genes on breast cancer prognosis using microarray data of 1809 patients. Breast Cancer Res. Treat. 2010, 123, 725-731. [CrossRef] [PubMed]

24. Nagy, A.; Munkárcsy, G.; Győrffy, B. Pancancer survival analysis of cancer hallmark genes. Sci. Rep. $2021,11,6047$.

25. Modhukur, V.; Iljasenko, T.; Lokk, K.; Metsalu, T.; Laisk-Podar, T.; Vilo, J. MethSurv: A web tool to perform multivariate survival analysis using DNA methylation data. Epigenomics 2018, 10, 277-288. [CrossRef] [PubMed]

26. Li, Y.; Ge, D.; Lu, C. The SMART App: An interactive web application for comprehensive DNA methylation analysis and visualization. Epigenetics Chromatin 2019, 12, 71. [CrossRef]

27. Wang, J.; Vasaikar, S.; Shi, Z.; Greer, M.; Zhang, B. WebGestalt 2017: A more comprehensive, powerful, flexible and interactive gene set enrichment analysis toolkit. Nucleic Acids Res. 2017, 45, W130-W137. [CrossRef]

28. Mi, H.; Muruganujan, A.; Thomas, P.D. PANTHER in 2013: Modeling the evolution of gene function, and other gene attributes, in the context of phylogenetic trees. Nucleic Acids Res. 2013, 41, D377-D386. [CrossRef] [PubMed]

29. Mi, H.; Ebert, D.; Muruganujan, A.; Mills, C.; Albou, L.P.; Mushayamaha, T.; Thomas, P.D. PANTHER version 16: A revised family classification, tree-based classification tool, enhancer regions, and extensive API. Nucleic Acids Res. 2021, 49, D394-D403. [CrossRef]

30. Mi, H.; Thomas, P. PANTHER pathway: An ontology-based pathway database coupled with data analysis tools. Methods Mol. Biol. 2009, 563, 123-140.

31. Benstead-Hume, G.; Wooller, S.K.; Downs, J.A.; Pearl, F.M.G. Defining Signatures of Arm-Wise Copy Number Change and Their Associated Drivers in Kidney Cancers. Int. J. Mol. Sci. 2019, 20, 762. [CrossRef]

32. Munro, D.; Ghersi, D.; Singh, M. Two critical positions in zinc finger domains are heavily mutated in three human cancer types. PLoS Comput. Biol. 2018, 14, e1006290. [CrossRef] [PubMed]

33. Li, J.; Song, L.; Qiu, Y.; Yin, A.; Zhong, M. ZNF217 is associated with poor prognosis and enhances proliferation and metastasis in ovarian cancer. Int. J. Clin. Exp. Pathol. 2014, 7, 3038-3047.

34. Zhang, C.; Xiang, T.; Li, S.; Ye, L.; Feng, Y.; Pei, L.; Li, L.; Wang, X.; Sun, R.; Ren, G.; et al. The novel 19q13 KRAB zinc-finger tumour suppressor ZNF382 is frequently methylated in oesophageal squamous cell carcinoma and antagonises Wnt/ $\beta$-catenin signalling. Cell Death Dis. 2018, 9, 573. [CrossRef]

35. Rousseau-Merck, M.F.; Koczan, D.; Legrand, I.; Moller, S.; Autran, S.; Thiesen, H.J. The KOX zinc finger genes: Genome-wide mapping of 368 ZNF PAC clones with zinc finger gene clusters predominantly in 23 chromosomal loci are confirmed by human sequences annotated in EnsEMBL. Cytogenet. Genome Res. 2002, 98, 147-153. [CrossRef]

36. Brooks, S.A.; Brannon, A.R.; Parker, J.S.; Fisher, J.C.; Sen, O.; Kattan, M.W.; Hakimi, A.A.; Hsieh, J.J.; Choueiri, T.K.; Tamboli, P.; et al. ClearCode34: A prognostic risk predictor for localized clear cell renal cell carcinoma. Eur. Urol. 2014, 66, 77-84. [CrossRef]

37. Brugarolas, J. Molecular genetics of clear-cell renal cell carcinoma. J. Clin. Oncol. 2014, 32, 1968-1976. [CrossRef]

38. Linehan, W.M.; Ricketts, C.J. The Cancer Genome Atlas of renal cell carcinoma: Findings and clinical implications. Nat. Rev. Urol. 2019, 16, 539-552. [CrossRef] 
39. Mehdi, A.; Riazalhosseini, Y. Epigenome Aberrations: Emerging Driving Factors of the Clear Cell Renal Cell Carcinoma. Int. J. Mol. Sci. 2017, 18, 1774. [CrossRef]

40. Peña-Llopis, S.; Vega-Rubín-de-Celis, S.; Liao, A.; Leng, N.; Pavía-Jiménez, A.; Wang, S.; Yamasaki, T.; Zhrebker, L.; Sivanand, S.; Spence, P.; et al. BAP1 loss defines a new class of renal cell carcinoma. Nat. Genet. 2012, 44, 751-759. [CrossRef]

41. Wagner, E.; Carpenter, P. Understanding the language of Lys36 methylation at histone H3. Nat. Rev. Mol. Cell. Biol. 2012, 13, 115-126. [CrossRef]

42. Dalgliesh, G.L.; Furge, K.; Greenman, C.; Chen, L.; Bignell, G.; Butler, A.; Davies, H.; Edkins, S.; Hardy, C.; Latimer, C.; et al. Systematic sequencing of renal carcinoma reveals inactivation of histone-modifying genes. Nature 2010, 463, 360-363. [CrossRef]

43. Pantuck, A.J.; Seligson, D.B.; Klatte, T.; Yu, H.; Leppert, J.T.; Moore, L.; O’Toole, T.; Gibbons, J.; Belldegrun, A.S.; Figlin, R.A. Prognostic relevance of the mTOR pathway in renal cell carcinoma: Implications for molecular patient selection for targeted therapy. Cancer 2007, 109, 2257-2267. [CrossRef]

44. Louie, B.H.; Kurzrock, R. BAP1: Not just a BRCA1-associated protein. Cancer Treat. Rev. 2020, 90, 102091. [CrossRef] [PubMed]

45. Lin, H.; Ma, N.; Zhao, L.; Yang, G.; Cao, B. KDM5c Promotes Colon Cancer Cell Proliferation through the FBXW7-c-Jun Regulatory Axis. Front. Oncol. 2020, 10, 535449. [CrossRef] [PubMed]

46. Schabath, M.B.; Giuliano, A.R.; Thompson, Z.J.; Amankwah, E.K.; Gray, J.E.; Fenstermacher, D.A.; Jonathan, K.A.; Beg, A.A.; Haura, E.B. TNFRSF10B polymorphisms and haplotypes associated with increased risk of death in non-small cell lung cancer. Carcinogenesis 2013, 34, 2525-2530. [CrossRef] [PubMed]

47. Provost, E.; Bailey, J.M.; Aldrugh, S.; Liu, S.; Iacobuzio-Donahue, C.; Leach, S.D. The tumor suppressor rpl36 restrains KRAS(G12V)-induced pancreatic cancer. Zebrafish 2014, 11, 551-559. [CrossRef]

48. Zheng, X.; Wu, K.; Liao, S.; Pan, Y.; Sun, Y.; Chen, X.; Zhang, Y.; Xia, S.; Hu, Y.; Zhang, J. MicroRNA-transcription factor network analysis reveals miRNAs cooperatively suppress RORA in oral squamous cell carcinoma. Oncogenesis. 2018, 7, 79. [CrossRef]

49. Khan, A.; Valli, E.; Lam, H.; Scott, D.A.; Murray, J.; Hanssen, K.M.; Eden, G.; Gamble, L.D.; Pandher, R.; Flemming, C.L.; et al. Targeting metabolic activity in high-risk neuroblastoma through Monocarboxylate Transporter 1 (MCT1) inhibition. Oncogene 2020, 39, 3555-3570. [CrossRef] [PubMed]

50. Lucarelli, G.; Loizzo, D.; Franzin, R.; Battaglia, S.; Ferro, M.; Cantiello, F.; Castellano, G.; Bettocchi, C.; Ditonno, P.; Battaglia, M. Metabolomic insights into pathophysiological mechanisms and biomarker discovery in clear cell renal cell carcinoma. Exp. Rev. Mol. Diagn. 2019, 19, 397-407. [CrossRef]

51. Wettersten, H.; Aboud, O.A.; Lara, P.N.J.; Weiss, R.H. Metabolic reprogramming in clear cell renal cell carcinoma. Nat. Rev. Nephrol. 2017, 7, 410-419. [CrossRef]

52. Netti, G.S.; Lucarelli, G.; Spadaccino, F.; Castellano, G.; Gigante, M.; Divella, C.; Rocchetti, M.T.; Rascio, F.; Mancini, V.; Stallone, G.; et al. PTX3 modulates the immunoflogosis in tumor microenvironment and is a prognostic factor for patients with clear cell renal cell carcinoma. Aging 2020, 12, 7585-7602. [CrossRef] [PubMed]

53. Beckermann, K.E.; Dudzinski, S.O.; Rathmell, J.C. Dysfunctional T cell metabolism in the tumor microenvironment. Cytokine Growth Factor Rev. 2017, 35, 7-14. [CrossRef]

54. Şenbabaoğlu, Y.; Gejman, R.S.; Winer, A.G.; Liu, M.; Van Allen, E.M.; de Velasco, G.; Miao, D.; Ostrovnaya, I.; Drill, E.; Luna, A. Tumor immune microenvironment characterization in clear cell renal cell carcinoma identifies prognostic and immunotherapeutically relevant messenger RNA signatures. Genome Biol. 2016, 17, 231. [CrossRef]

55. Siska, P.J.; Beckermann, K.E.; Mason, F.M.; Andrejeva, G.; Greenplate, A.R.; Sendor, A.B.; Chiang, Y.C.J.; Corona, A.L.; Gemta, L.F.; Vincent, B.G.; et al. Mitochondrial dysregulation and glycolytic insufficiency functionally impair CD8 T cells infiltrating human renal cell carcinoma. JCI Insight 2017, 2, e93411. [CrossRef] [PubMed] 